Portland State University

PDXScholar

1989

\title{
The Effects of Bandwidth Limiting Tuning Elements on a Synchronously Pumped Mode-Locked Dye Laser
}

Bahram Zandi

Portland State University

Follow this and additional works at: https://pdxscholar.library.pdx.edu/open_access_etds

Part of the Electrical and Computer Engineering Commons Let us know how access to this document benefits you.

Recommended Citation

Zandi, Bahram, "The Effects of Bandwidth Limiting Tuning Elements on a Synchronously Pumped ModeLocked Dye Laser" (1989). Dissertations and Theses. Paper 1129.

https://doi.org/10.15760/etd.1128

This Dissertation is brought to you for free and open access. It has been accepted for inclusion in Dissertations and Theses by an authorized administrator of PDXScholar. Please contact us if we can make this document more accessible: pdxscholar@pdx.edu. 
THE EFFECTS OF BANDWIDTH LIMITING TUNING ELEMENTS ON A SYNCHRONOUSLY PUMPED MODE-LOCKED DYE LASER

by

BAHRAM ZANDI

A dissertation submitted in partial fulfilment of the requirements for the degree of

DOCTOR OF PHILOSOPHY

in

ELECTRICAL AND COMPUTER ENGINEERING

Portland State Univeralty

(C) 1989 
TO THE OFFICE OF GRADUATE STUDIES:

The memberg of the committee approve the dissertation of

Bahram Zandi pregented May 1, 1989.

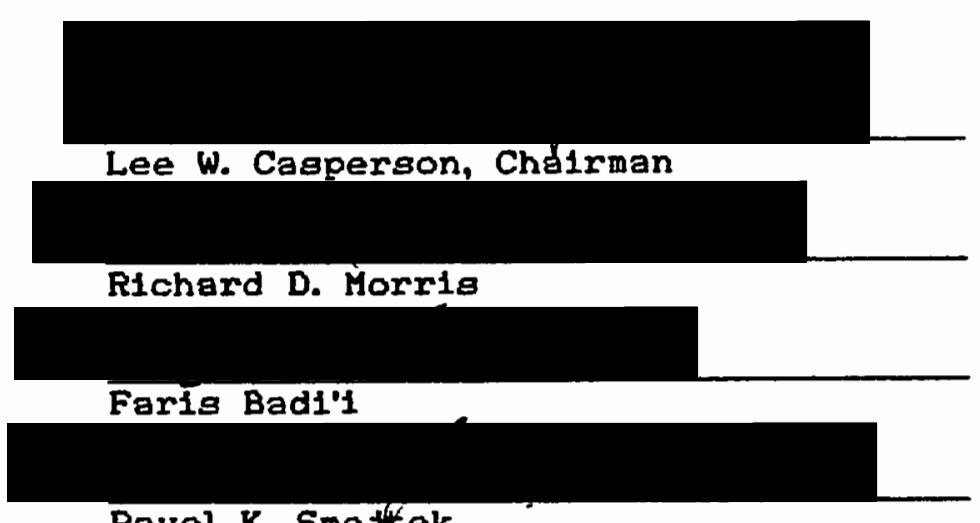

Pavel K. Smeftek

\section{APPROVED:}

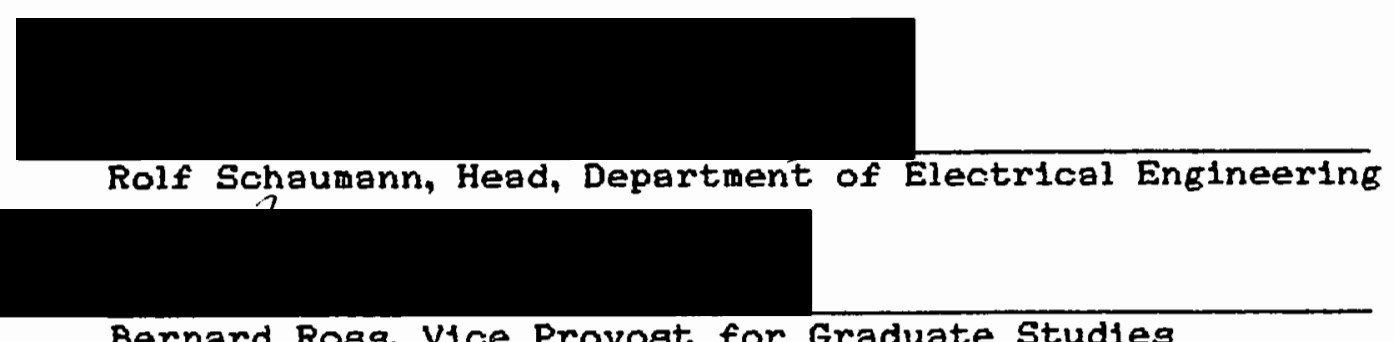

Bernard Ross, Vice Provost for Graduate Studies 
AN ABSTRACT OF THE DISSERTATION OF Bahram Land for the Doctor of Philosophy in Electrical and Computer Engineering presented May 1, 1989.

Title r The Effects of Bandwidth Limiting Tuning Element a on a Synchronously Pumped Mode-Locked Dye Laser

APPROVED BY MEMBERS OF THE DISSERTATION COMMITTEE:

Lee Carperuon

Lee w. Casperson, Chairman

Qritad A. Morn is

Richard D. Morris

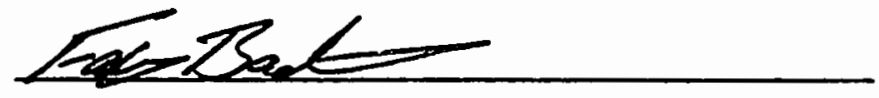

Farts Bad1'1

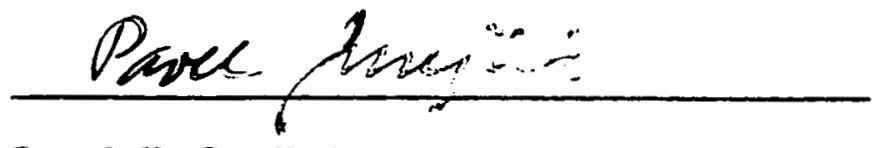

Ravel K. Sa ejtek

In this study an improved description of the bandwidth 


\section{AN ABSTRACT OF THE DISSERTATION OF Bahram Zandi for the Doctor of Phllogophy in Electrical and Computer Englneering presented May $1,1989$.}

Titles The Effects of Bandwidth Limiting Tuning Elements on a Synchronously Pumped Mode-Locked Dye Laser

APPROVED BY MEMBERS OF THE DISSERTATION COMMITTEE:

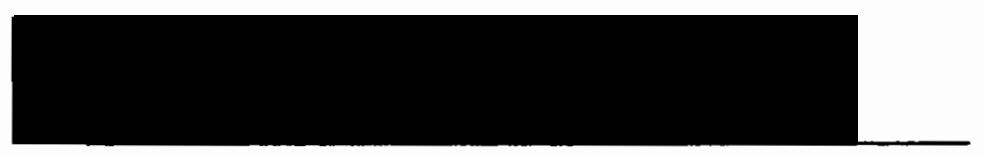

Lee w. Casperson, Chairman

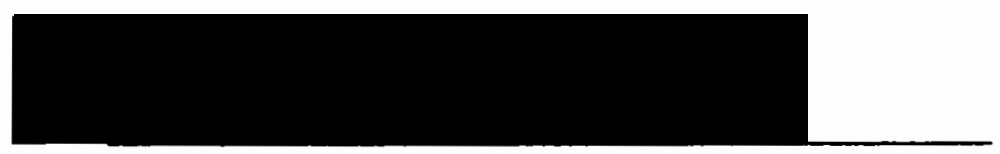

Richard D. Morris

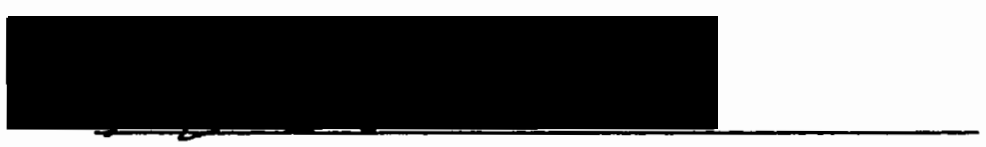

Faris Badi'1

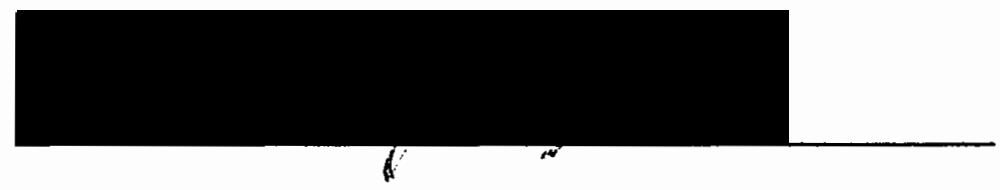

Pavel K. Smeftek

In this study an improved description of the bandwidth 
limiting tuning filters has been introduced into a semiclassical model for a synchronouly pumped mode-locked dye laser system. This model is an improvement over the traditional rate equation approach since tt has detalled representations of the energy levels, rotational distribution, and coherence effects. The new set of equationg has been solved numerically using the best avallable values for the various parameters, and autocorrelations have been computed for a range of different length detuning and bandwidth limiting elemerits. In the experiments, an acoustooptically mode-locked argon ton laser is used to synchronously pump a rhodamine $6 G$ dye laser. To study the tuning effects, two and three plate birefringent filters and a tuning wedge have been used. Transmission and bandwidth of these filters have been carefully measured as a function of the length detuning. The autocorrelated pulses have been measured as a function of the length detuning. The experimental pulse shapes agree closely with the theoretical solutions for all valueg of detuning and filter bandwidth. Through a sensitivity analysis, it is shown how this model can be used to select values for the transmission and the bandwidth of the filter to obtain optimum pulse characteristics. 
To my mother and to the memory of my father 


\section{ACKNOWLEDGEMENTS}

I have had many great teacherg to whom I am indebted. I would particularly like to thank my advieer, Profesgor Lee w. Casperson, who taught me all that I know about lasera. I would also like to thank all my family and friends for their support, especially Karen Nordgren. And finally I would like to thark my colleague Dr. Duncan L. MacFarlane for the valuable discusgiong we had. 
TABLE OF CONTENTS

PAGE

ACFNOWLEDGEMENTS $\ldots \ldots \ldots \ldots \ldots \ldots \ldots \ldots \ldots \ldots \ldots \ldots \ldots \ldots \ldots$ i $i \ldots \ldots$

LIST of TABLES $\ldots \ldots \ldots \ldots \ldots \ldots \ldots \ldots \ldots \ldots \ldots \ldots \ldots \ldots$

LIET OF FIGUREE $\ldots \ldots \ldots \ldots \ldots \ldots \ldots \ldots \ldots \ldots \ldots \ldots \ldots \ldots$. . . . . . . . . .

CHAPTER

I INTRODUCTION $\ldots \ldots \ldots \ldots \ldots \ldots \ldots \ldots \ldots \ldots \ldots \ldots$

II THEORY $\ldots \ldots \ldots \ldots \ldots \ldots \ldots \ldots \ldots \ldots \ldots \ldots$

The sem1clagalcal Approach...............

Derlvation of the Inhomogeneoug Wave Equation....7

Quantum Mechanical Derivation of the Polarization. 9

Inclualon of the Bandwidth Limiting Element..... 17

The Steady State Limit and Normalization...... ¿e

Synchronoug pumping.................. 28

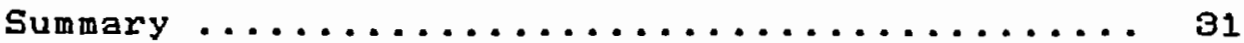

III EXPERTMENT $\ldots \ldots \ldots \ldots \ldots \ldots \ldots \ldots \ldots \ldots \ldots \ldots \ldots \ldots \ldots$

Argon Lagex Pump ..................... 35

The Eynchranougly Pumped Dye Lager ......... 36 


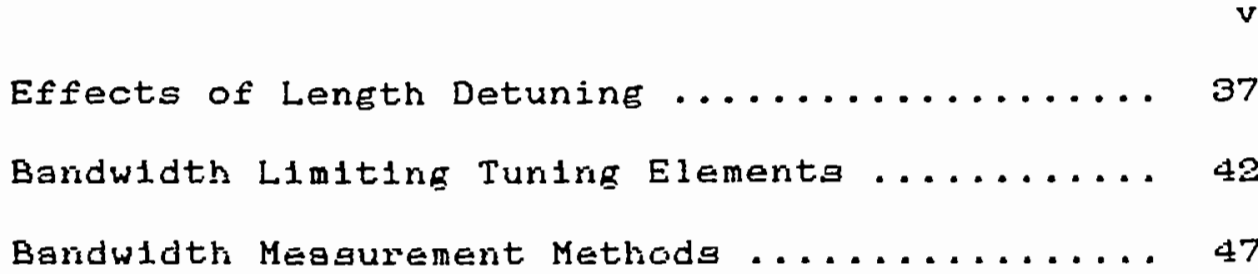

IV RESUltS AND CONCLUSIONS .............. 51

$\checkmark$ EURTHER NUMERICAL ETUDIES .............. Sensitivity Analygis (Englneering Degign)..... 61 Suggestiong For Further Studieg .......... 82

REFERENCES CITED ........................ 87

\section{APPENDICES}

A Mechanism of Formation of a Picosecond Pulse.... 69

B Measuring Picosecond Pulses .............. 72 


\section{LIST OF TABLES}

TABLE

PAGE

I PARAMETERS $\ldots \ldots \ldots \ldots \ldots \ldots \ldots \ldots \ldots \ldots \ldots \ldots \ldots \ldots$ 


\section{LIST OF FIGURES}

FIGUKE

PAGE

1. Eremey levela of the dye lager model.............. 1e

2. Lorentzian 1 ineshape $\ldots \ldots \ldots \ldots \ldots \ldots \ldots \ldots \ldots \ldots \ldots$

9. Sample theoretical plot ..................

4. Our sync. pumped modelocked dye laser system .....4 41

5. Dye laser closeup $[31] \ldots \ldots \ldots \ldots \ldots \ldots \ldots \ldots \ldots$

B. Tuning wedge $[31] \ldots \ldots \ldots \ldots \ldots \ldots \ldots \ldots \ldots$

7. Birefringent filter [32].................48

8. Three plate transmigsion curve ..............49

9. Two plate transmisston curve ...............50

10. Tuning wedge trangmisalon curve ...............50

11. Autccorrelation of the output pulse for the $\exists$ plate

filter with a) $5 \mu$ and b) $18 \mu$ length detuning...55

12. Autocorrelation of the output pulge for the 3 plate

fllter with a) $48 \mu$ and b) $71 \mu$ length detuning ..54

13. Autocorrelation of the output pulge for the 2 plate filter with a) $3 \mu$, b) $8 \mu$ and c) $11 \mu$ length

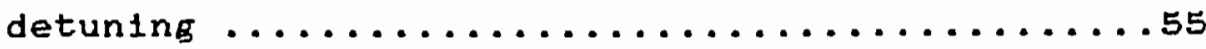


14. Autocorrelation of the output pulge for the tuning wedge with a) $2 \mu$, b) $B \mu$ and c) $21 \mu$ length detuning.........................

15. Theoretical line and experimental barg for the 9 plate filter .....................

1E. Theoretical line and experimental barg for the 2 plate filter ......................

17. Theoretical line and experimental bars for the tun1ng wedge .......................

18. Pulgewidth vg $\beta \ldots \ldots \ldots \ldots \ldots \ldots \ldots \ldots \ldots \ldots \ldots$

19. Peak intensity vs $\beta \ldots \ldots \ldots \ldots \ldots \ldots \ldots \ldots \ldots \ldots$

20. Pulsewidth vs bandwidth $\ldots \ldots \ldots \ldots \ldots \ldots \ldots \ldots \ldots$

21. Peak intengity vs bandwidth ...............66

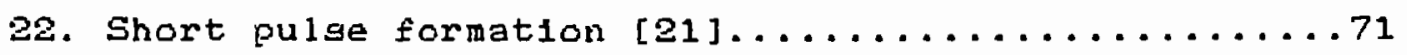

23. Schematic of Spectra-Physicg model 409 autocorrelator $[29] \ldots \ldots \ldots \ldots \ldots \ldots \ldots \ldots \ldots \ldots \ldots \ldots \ldots$ 
CHAPTER I

INTRODUCTION

Syrchronous optical pumping le one of the most popular methods of producting tunable ultrashort pulses, which have many practical applications. The gain of an active medium is modulated at a frequency equal to or a multiple of the roundtrip frequency of the laser resonator. The output is a train of pulses, synchronoug with the pump pulse, but with much smaller durations. These pulses have numerous applicationg, including for example, high speed spectroscopy. The output of an actively mode-locked Ion laser which is logs modulated is used to pump a dye lager which is thereby actively mode-locked through gain modulation and produces plcosecond pulses.

Previous studies of synchronously pumped mode-locked lager aystems have uged rate equation analyses, which describe temporal changeg of the atomlc populationg and the light energy (or the number of photong), neglecting the phage of the light and the atomic polarization. The first approaches used a two level model [1-2], and later studies Introduced many level model approaches [3-19,21]. The first sem1-classical method was Introduced by Casperson in 1983 [20] which is the starting point for this disgertation. To achieve tunability several methods 
have been used. Organtc dye lasers are popular because of the high repetition frequency of the pulses, stability of amplitude and wide wavelength tuning range due to the wide fluorescence of an organic dye. The dye laser was discovered in 1965 by $P$. Sorokin and coworkers at the IBM laboratorles. With dye lagers, synchronous pumping has been achleved either by a continuous pulae train or by a finite pulge train uging argon, krypton, or YAG:Nd ${ }^{3+}$ lasers for the pump. Besides dye lasers, other active media used are color-center crystal lasers, semiconductor crystal lasers, and lasers utilizing nonlinear optical active media such as optical parametric oscillatorg and Raman lagers [21]. Also combined systems of synchronously pumped tunable picosecond lasers have been used including: active-passive mode locking, synchronously pumped dye lasers with hard exctation, synchronous lasing of tunable lasers at many frequencies, use of binary mixtures of dye solutions, and synchronously pumped tunable lasers with cavity dumping [21].

This dissertation uses the semiclassical Maxwell-schrodinger approach which has several advantages over the previous rate equation studies. First, it considers vectors for the electric fields, therefore enabling the inclusion of an anisotropic orientational distribution. Second, it includes the phase memory time of the molecular wave functions and a finite vibrational relaxation time in the lower electronic state of the dye laser trangition.

Laser power and temporal characteristics are determined by 
the Interaction between the light and the atoms under the Influence of pumping and relaxation. There are three different approaches in treating the laser theoretically: via rate equations, through a semiclasglcal theory, or using a full quantum mechanical theory. These three formulations are not independent; the rate equation approsch is an approximation to the eemiclaseical approach, which in turn is an approximation to the quartum mechanical approach. The classical colliaion picture that 1a behind the almple rate equation approach yields accurate power and threshold predictions and provides a reasonable model for some nonlinear and time dependent effects. Since the rate equations describe temporal changes of atomic populations and light energy or the number of photons, neglecting the phase of the light and atomic polarization, they break down when ensemble effects become important. At the other extreme is the quantum mechanical approach. In the full quantum mechanical theory, both the atoms and the light are quantized and treated quantum mechanically uaing density matrices [22]. The quantum mechanical approach is typically used when the problem involveg quantum nolse since it rigorously treats spontaneoug emisgion. It is also esgential in answering questions of photon statistics and fleld bulldup from vacuum. A disadvantage of this treatment is that it is too cumbersome.

In the aemiclassical theory light is treated as classical electromagnetic waves described by Maxwell equations, while the ensemble of atoms that interact with the electromagnetic field 
are described quantum mechanically. Semiclassical effects form a middle ground between the simple rate equation plcture and the extensive quantum mechanical theory. Semiclagsical theory must be used to describe certain "coherent" interactions between light and matter such $a s$ photon echoes, self induced transparency, or spontaneoug pulsations from Xe lasers. These effects are really the macroscopic manifestations of a quantum mechanical ensemble and the distribution of information, or "coherence" of that ensemble is inherent in those phenomena.

These coherence effects typically manifest themselves on short time scales. One can probably neglect conerence effects if the intensity changes on a time scale that is long with respect to the coherence time. Since the mode-locked laser pulses studied in this work are only a few picoseconds long and the coherence time of the dye laser medium is $5 \times 10^{-14}$ sec., it is important to include these coherent effects. Few studies of mode-locked lasers, however, are complete enough to include those effects.

Convergely, we are interested more in the shape of the ideal steady state pulse and not in its buildup from nolse. Consequently, in this study we adopt the semiclassical approach and consider the model of a synchronously pumped mode-locked dye laser system with an intracavity tuning element.

The order for the organisation of the chapters is as following: In Chapter II, we present the derivation of our model for a synchronously pumped mode-locked dye laser with a 
bandwidth limiting tuning element. Chapter III describes the design of our experiments. Chapter IV deals with our results and conclualons. Chapter $V$ has further numerical studies and sensitivity analysis. 
CHAPTER II

THEORY

THE SEMICLASSICAL APPROACH

\begin{abstract}
We now detall further, the components of a semiclagsical theory. A semiclassical treatment does not use the quantized radiation fleld but treats the electromagnetic field as a clasaical, external force acting on the atomic aystem providing the gain mechanism. The radiation field induces an electric dipole moment in the material which in turn is used in the classical Maxwell equations to calculate the effect of the gain medium on the field.

A classical electromagnetic field is governed by Maxwell's equations. We demand that the field be self-consistent: the field $E$ that induces the polarization of the active medium must be equal to the resulting field $E^{\prime}$. The self consistent loop of the gemiclassical formalism is as follows:
\end{abstract}

$E(r, t) \rightarrow$ Quantum Mechanics $\rightarrow$ Statistical Summation $\rightarrow P(r, t) \rightarrow$ Maxwell's Equations $\rightarrow E^{\prime}(r, t)$

Here the field induces electric-dipole moments in the medium 
according to the laws of quantum mechanics. The dengity matrix is used to facilitate the gtatistical summationg that govern the Interaction of a single particle with an externally applied electric field involved in obtaining the macroscoptc polarization of the medium for the individual dipole moments. The density matrix formaliam is an extengion of quantum mechanics which Includes statiotical engemble averaging of incompletely known phyelcal aystems. The macroscopic polarization then becomes the driving force in the Haxwell wave equation that produces the fleld.

In the following sections, we preaent the derivation of our model for a aynchronously pumped mode-locked dye lager with a bandwidth limiting tuning element.

\section{DERIVATION OF THE INHOMOGENEOUS WAVE EQUATION}

Starting from the clagatcal Maxwell equatione:

$\mathbf{x} \mathbf{H}=\delta \mathrm{D} / \delta t+\mathbf{J}$

(1)

$\mathbf{F} \mathbf{E}=-\delta \mathrm{B} / \delta \mathrm{t}$

(2)

- $\mathbf{B}=0$

(1)'

D. $=\rho$

(2)'

where $E$ is the electric field, $D$ is the electric flux dengity (displacement), H is the magnetic fleld, B is the magnetic flux density, $J$ is the electric current dengity, and $p$ is the charge 
density. For the displacement vector $\mathbf{D}$ we have:

$\mathbf{D}=e_{\mathrm{O}} \mathbf{E}+\mathbf{P}$

where $P$ is the polarization vector that represents the material Interaction that will be the toplc of aection c. In the absence of magnetic polarization we have for the magnetic induction vector:

$\mathbf{B}=\mu_{0} \mathbf{H}$

Using the constitutive relations (3) and (4), we can write Maxwell's equations as:

$\nabla \mathbf{x}=e_{0} \delta E / 8 t+\delta P / 8 t$

(5)

$\nabla \mathbf{X}=-\mu_{0} \delta H / \delta t$

(8)

Taking the curl of eq.(6) and using eq.(5) we obtains

$\nabla x(\nabla \times E)=-\epsilon_{O} \mu_{O} \delta^{2} E / \delta t^{2}-\mu_{O} \delta^{2} P / \delta t^{2}-\mu_{O} \sigma \delta E / \delta t$

(7)

Using the vector 1dentity:

$\nabla \times(\nabla \times E)=\nabla(\nabla . E)-\nabla^{2} E$

(8)

and by considering the charge free limit, $\nabla=0$, we obtain an 
inhomogeneous wave equation for the electric fleld vector

$\nabla^{2} E-e_{O} \mu_{O} \delta^{2} E / \delta t^{2}-\mu_{O} \sigma \delta E / \delta t=\mu_{O} \delta^{2} P / \delta t^{2}$

This equation will give us the fleld $E$ once we know the polarization $P$, and may be viewed as complementary to the Schrodinger equation.

Now we aggume a plane wave solution for our gignal fleld in the $z$ directions

$E_{g}(z, t)=1 / 2 E_{g}(z, t) \exp \left[1\left(k_{g} z-\omega_{g} t\right)\right]+c . c$.

Recall that the polarization vector $P$ must be obtalned from the quantum theory of the atomic system. This then is our next task.

\section{QUANTUM MECHANICAL DERIVATION OF THE POLARIZATION}

The macroscopic polarization has 1ts orfgin in the microscopic response of a molecule bathed in an electric field [Debye]. We chose to treat this problem in a quantum mechanical framework and thus recall the time dependent schrodinger equation which may be written agt

$$
H \oplus(r, t)=1 h \delta \Phi(r, t) / \delta t \quad(10)
$$

where $H$ is the Hamiltonian operator, 
Here $V$ is the potential energy of the particle's immediate environment plus a perturbation due to the field.

From the works of Lamb [22], we may now assume a solution form or a sum of orthonormal solutiong:

$\Phi(r, t)=\varepsilon c_{n}(t) u_{n}(r)$

(13)

Here $u_{n}(r)$ are solutions to the time independent, unperturbed Schrodinger wave equation. Also uging ensemble averaging and Introducing the density matrix:

$p_{\text {nm }}=c_{m}^{*} c_{n}$

(14)

we obtain:

$\delta \rho_{n m} / \delta t=1 / K \Sigma\left(\rho_{n j} H_{m j} *-\rho_{j m} H_{n j}\right)$

(15)

or, introducing the commutator notation

$\delta \rho / \delta t=1 / K[\rho, H]$

(18)

Equation (16) is the equation of motion for the density matrix. It relates the time rate of change of the density 
matrix element to a Hamiltonian, which for our problem las

$H=H_{O}+H^{\prime}=H_{O}-\mu E(t)$

Here, $H^{\prime}$ is the time dependent perturbation that the molecule feels when subjected to an electric fleld $\mathbf{E}(t)$. The strength of this perturbation 1 s proportional to the dipole moment operator H, which in turn 18 proportional to the charge per volume $e$,
$\mu=\mathbf{e r}$
(17)

Our goal ia the macroscopic polarization of equation (9), which we define as the dipole moment per unit volume,
$\mathbf{P}=\mathbf{N}\langle\boldsymbol{\mu}\rangle$
(19)

where $\mathrm{N}$ is the number of atoms per volume and $\langle\mu\rangle$ is the expectation value of the dipole moment.

For a two level atomic energy aystem, we can write the set of density matrix equations as [20]s

$\delta p_{21} / \delta t=-1 w_{0} p_{21}-1 / K \mu_{21} \cdot E\left(p_{22}-\rho_{11}\right)$

$\rho_{12}=\rho_{21}^{*}$

$d / d t\left(p_{22}-p_{11}\right)=$ 
$=-21 E / K\left(\mu_{12} P_{21}-\mu_{21} P_{12}\right)$

(23)

Coherent equationg (21-23) represent the stimulated (emiggion and absorption) response of the atom to an applied, time dependent field. Equation (23) Boverng the population difference while equations (21) and (22) determine the polarization.

Following from [20], we consider a four level model for our dye laser system as in Figure 1. When the dye is excited by an external source of light, it emits radiation at longer wavelength (fluoresces), absorbing a photon at the excltation wavelength and exitting a photon at the fluorescenge wavelength. The energy difference between the absorbed and emitted photon is accounted for by a nonradiative trangition in the dye which transforms into heat.

Therefore we can write the density matrix equations for this four level atomic energy system as:

$$
\begin{aligned}
& \delta \rho_{00} / \delta t=-i / K\left(\rho_{03} \mu_{30}-\rho_{30} \mu_{O 3}\right) \cdot E_{p}+\rho_{11} / T_{1} \\
& \delta \rho_{33} / \delta t=i / K\left(\rho_{03} \mu_{30}-\rho_{30} \mu_{03}\right) \cdot E_{p}-\rho_{33} / T_{3} \\
& \delta \rho_{22} / \delta t=-i / K\left(\rho_{21} \mu_{12}-\rho_{12} \mu_{21}\right) \cdot E_{S}-\rho_{22^{\prime}} / T_{2}+\rho_{33^{\prime}} / T_{3} \\
& \delta \rho_{11} / \delta t=i / K\left(\rho_{21} \mu_{12}-\rho_{12} H_{21}\right) \cdot E_{s}-\rho_{11} / T_{1}+\rho_{22^{\prime}} T_{2} \\
& \delta \rho_{30} / \delta t=-i \omega_{P O} \rho_{30}+i / K\left(\rho_{00}-\rho_{33}\right) \mu_{30} \cdot E_{p}-\rho_{30^{\prime}} / T_{P}
\end{aligned}
$$


$\delta \rho_{21} / \delta t=-i \omega_{s 0} \rho_{21}-i / K\left(\rho_{22}-\rho_{11}\right) \mu_{21} \cdot E_{s}-\rho_{21} / T_{s}$

$\rho_{03}=\rho_{30}{ }^{*}$

$\rho_{12}=\rho_{21}^{*}$

The $E_{p}$ and $E_{E}$ are the pump and algnal electric fields, wpo and $\omega_{s} 0$ are the center frequencles of the pump and algnal transitions, $T_{p}$ and $T_{B}$ are the coherence times of the off diagonal matrix elements and the $\mu^{\prime} s$ are the matrix elements of the dipole moment operator. Note also that we have phenomenologically included the spontaneous decay in terms of the lifetimes $T_{1}, T_{2}, T_{3}$.

Thus our semiclaselcal model for a dye laser medium without a fllter, consists of three density matrix equations:

$\delta p_{22} / \delta t=\eta_{s} E_{g} \times / h-p_{22} / T_{2}+T_{p} /\left.\mu_{p}\right|^{2} E_{p}{ }^{2}{ }^{2} / 2 H^{2}$

$\delta p_{11} / \delta t=-\eta_{s} E_{s} x / h-p_{11} / T_{1}+p_{22} / T_{2}$

$\delta \eta_{s} / d t=1\left(\omega_{s}-\omega_{s}\right) \eta_{s}-\left(p_{22}-p_{11}\right) / \mu_{s} / 2 E_{s} x-\eta_{s} / T_{s}$

and one Maxwell equation for the electric fleld. In eection (c) we derived the wave equation and suggegted a plane wave 
solution. Now we substitute the polarizations

$P_{s}=\int n(\theta, \phi)\left(\rho_{21} \mu_{12}+\rho_{12} \mu_{21}\right) d \Omega$

with the propagation constant,

$\mathbf{k}_{s}{ }^{2}=$ Hew $_{s}{ }^{2}$

and phase velocitys

$v=(\mu e)^{-1 / 2}$

and where higher order space and time derivatives of slowly varying quantities are neglected. Also we have used the rotating wave approximation which limits the overall response of the atom to one near regonance. Therefore we obtain:

$\delta E_{s} / \delta z+1 / v \delta E_{\sigma} / \delta t+\alpha / 2 E_{0}=-\mu \omega_{s}{ }^{2} / k_{s} \int \eta_{s} x d x$

where the $\rho$ 's are population, $E_{p}$. is the pump field, $E_{S}$ is the signal field, $T_{s}$ is the coherence time, $\mu$ is the dipole moment, $\omega$ is the signal frequency and $a$ is the logs term. Also we have introduced:

$\eta(\theta, \Phi, z, t)=\rho \mu$

(29) 
$\theta$ measures the angle of a class of pump dipoles with reapect to the $z$ axis.

$x=\cos \theta$, is the molecular distribution factor. It has been shown that the inclusion of $x$ is necessary for a rigorous analyaig and for better agreement with the experiment [20]. For unidirectional distribution we can assume.
$\mathbf{x}=1$
(30)

The limit of $T_{S}=0$, represents the rate equations approximation. 


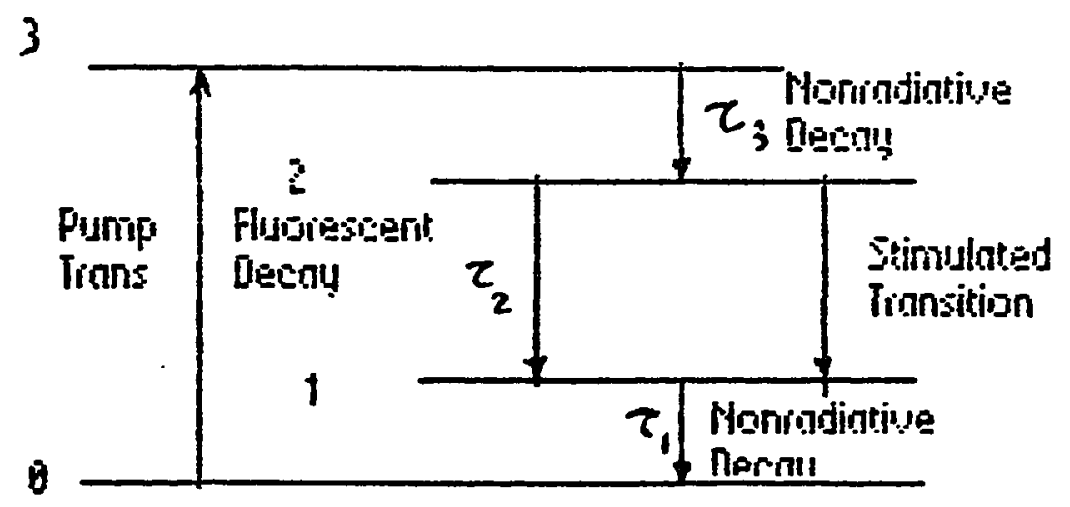

Figure 1. Energy levels of the dye lager model. 
INCLUSION OF THE BANDWIDTH LIMITING ELEMENT

To Incorporate the bandwidth limiting filter in our dye laser model, we agsume that the fllter is a two level negstive absorber (amplifier), consisting of a bandwidth limiter and an attenustor, and in anslogy to resl atomic trangitions, it has a Lorentzian lineshape (see Figure 2 ). In a block diagram, using analog elements, we can show it as a linear bandpass filter.

Since for the fliter, we assume a two level model. therefore the same dye lager equations $(25-28)$ can be adapted with:

$$
\mathbf{E}_{\mathrm{p}}=0
$$

and

$$
\begin{aligned}
& T_{1} \rightarrow \infty \\
& \delta \rho_{22 f} / \delta t=\eta_{f} / \text { } E_{S}-\rho_{22 f} / T_{f} \\
& \delta \rho_{11 f} / \delta t=-n_{f} / h E_{S}+\rho_{22 f} / T_{f} \\
& \delta n_{f} / \delta t=1\left(\omega_{s}-\omega_{f O}\right) n_{f}-n_{f} / T_{f}-1\left(\rho_{22 f}-\rho_{11 f}\right) \mid \mu_{f} / 2 E_{s} / 2 k
\end{aligned}
$$

where the subscript $f$ is for the filter. We note that eq. 31 and 32 are not linearly independent, specifically,

$$
\delta p_{11 f} / \delta t=-\delta p_{22 f} / \delta t
$$


or:

$$
\begin{aligned}
& \delta\left(\rho_{11 f}+\rho_{22 f}\right) / \delta t=0 \\
& \rho_{11 f}+\rho_{22 f}=\text { constant }
\end{aligned}
$$

where the arbitrary constant of integration in eq. (38) is chosen to be unity in keepline with the probabilistic interpretation of the density matrix.

Dividine $\eta_{f}$ into real and imaginary parts, we have:

$$
\eta_{f}=\eta_{f}^{\prime}+1 n_{f}
$$

substituting into eq. (38) we have:

$$
\delta n_{f} / \delta t=-n_{f}\left(\omega_{\delta}-\omega_{f O}\right)-n_{f}^{\prime} / T_{f}
$$

$\delta n_{f}^{\prime} / \delta t=n_{f}^{\prime}\left(\omega_{s}-\omega_{f o}\right)-n_{f}^{\prime \prime} T_{f}-\left|\mu_{f}\right| 2_{E_{g}}\left(2 p_{22 f}-1\right) / 2 k$ (38)

To solve for the frequency lineshape for the response of the atoms in steady state, we equate the time derivatives to zero. From eq. (31)z

$$
P_{22 f}=T E_{S} \eta_{f} / K \quad(40)
$$

From eq. (38): 


$$
n_{f}=-\eta_{f}^{*} / T_{f}\left(\omega_{s}-\omega_{f O}\right)
$$

From eq. (39):

$n_{f}^{\prime}\left(\omega_{s}-\omega_{f o}\right)=$

$=-\eta_{f}^{\prime} / T_{f}^{2}\left(\omega_{s}-\omega_{f O}\right)+\left|\mu_{f}\right|^{2} E_{s} / 2 K\left[-2 E_{s} T_{f} n_{f}^{\prime} / K T_{f}\left(\omega_{s}-\omega_{f O}\right)-1\right]$ (42)

solving for $\eta_{f}^{\prime}$

$n^{\prime}=$

$=-E_{g}\left|\mu_{f}\right|^{2 / 2 K} T_{f}^{2\left(\omega_{g}-\omega_{f O}\right) /\left[1+T_{f}{ }^{2}\left(\omega_{s}-\omega_{f O}\right)^{2}+\left|\mu_{f}\right|^{2} E_{g}{ }^{2} T_{f} f_{f} / K^{2}\right]}$ (43)

Similarlyz

$n^{\prime \prime}=$

$=E_{g} \mid \mu_{f}{ }^{2} T_{f} / 2 K\left[1+T_{f}{ }^{2}\left(\omega_{g}-\omega_{f O}\right)^{2}+\left|\mu_{f}\right|^{2} E_{s}{ }^{2} T_{f} f_{f} / K^{2}\right]$

and from (40):

Pz2f $=$

$=T_{f} E_{s}^{2} T_{f}\left|\omega_{f}\right| 2 / 2 K^{2}\left[1+T_{a}{ }^{2}\left(\omega_{s}-\omega_{f O}\right)^{2}+\left|\mu_{f}\right|^{2} E_{s}{ }^{2} T_{f} T_{f} / K^{2}\right]$

Now we have a saturating Lorentzian. But a linear filter does not saturate, therefore to have nonsaturating linear filter, 
20

we need to haves

$T_{f} \rightarrow 0$ which leads to $\rho_{22} \ll$

Therefore our equations (31), (38) and (39) become:

$\delta_{22 f^{\prime}} / 8 t=n_{f}^{m} / K E_{s}-p_{22 f^{\prime T}}$

(46)

$\delta \eta_{f}^{\prime} / \delta t=-\eta_{f}^{\prime}\left(\omega_{s}-\omega_{f o}\right)-\eta_{f}^{\prime} / T_{f}$

$\delta \pi_{f}^{m} / \delta t=\eta_{f}^{\prime}\left(\omega_{g}-\omega_{f 0}\right)-\eta_{f} / T_{f}+\left|\mu_{f}\right| z_{E_{g}} / 2 K$

(48)

Solving for steady state we obtain:

$P_{22 f}=T_{f}\left(E_{s} / K\right) \eta_{f}$

(49)

$n_{f}=-n_{f}^{\prime} / T_{f}\left(\omega_{g}-\omega_{f 0}\right)$

(50)

Thus,

$\boldsymbol{n}_{\mathbf{f}}=$

$=\left|\mu_{f}\right|^{2} E_{s} T_{f}^{2}\left(w_{s}-w_{f O}\right) / 2 K\left[1+T_{f}^{2}\left(w_{s}-w_{f O}\right)^{2}\right]$

(51)

$n^{\prime \prime}=$

$=\left|\mu_{f}\right|^{2} E_{S} T_{f} / 2 K\left[1+T_{f}{ }^{2}\left(w_{g}-w_{f O}\right)^{2}\right]$

(52)

$\rho_{22 f}=$

$=\left.\mu_{f}\right|^{2} E_{S}{ }^{2} T_{f} f_{f} / 2 K^{2}\left[1+T_{f}{ }^{2}\left(w_{s}-w_{f O}\right)^{2}\right]$

(53) 
In equation (52), we observe the unsaturating Lorentzian 11neshape.

Since we are interested in the temporal response of the filter at line center we set:

$$
w_{s}=w_{f o}
$$

then,

$$
n_{1}^{\prime}=0
$$

and our filter response equation becomes:

$$
\delta \pi_{f} / \delta t=-\pi_{f} / T_{f}+\left|\omega_{f}\right|^{2} E_{E^{\prime}} / 2 k
$$

Equation (58) is the only relevent equation from this set, because the field can only interact with $n_{f}$ and pezf is decoupled from this equation. We note that eq. (48) is the same equation as eq. (56). 
To couple $\eta_{h}$ into the field equation for our model, we need to define the parameters characterising our filter amplifier and attenuator. As they are presented in Figure 2 , we have shown our bandwidth limiting filter in two partas Firgt, as an amplifier (abaorber) and second, as an attenuator. This formaliam is restrictive. We need to select a many parameters for our model of the filter as possible, while keeping efficiency. There are three olgnificant parametera:

1. Bandwidth of the filter at fullwidth half maximum.

2. Maximum transmission of the filter.

3. Minimum transmission of the filter.

From Figure 2 , for linewidth correction due to finite interaction length, we have:

$$
\begin{aligned}
& \text { Amplifier gain }=T_{\max } / T_{\min } \\
& \text { Attenuator gain }=T_{\min } \\
& I_{\text {out }}=I_{\text {in }} e^{g z} \\
& I_{\text {out }}-I_{\text {In }}=I_{\text {In }}\left(e^{E z}-1\right) \\
& e^{E(0) z}=I_{\max } / T_{\min }
\end{aligned}
$$




$$
g(0) z=\ln \left(T_{\max } / T_{\min }\right) \quad(58)
$$

We demand that the difference (57) drop to $1 / 2$. Therefore:

$$
1 / 2=\left[e^{\left.g(y / s) z_{-1}\right]},\left[e^{\left.g(0) z_{-1}\right]}(58-a)\right.\right.
$$

According to reference [24] on spectral Narrowing in High-Gain Lasers, $y$ is the normalized frequency in homogeneously (Doppler) broadened medium and is shown as:

$$
y=2\left(\nu-\nu_{0}\right) / \Delta \nu_{h} \quad(58-b)
$$

For homogeneous broadening we assume a Lorentzian response:

$$
g(y)=g(0) /\left[1+y^{2}\right]
$$

Substituting in $(58-a)$ :

$1 / 2=\left[e^{g(0) z / 1+y} y_{1 / 2}^{2}-1\right] /\left[e^{E(0) z}-1\right](58-c)$

Then solving for $y_{1 / 2}$ in $(58-c)$, we can find $\Delta \nu$ from $(58-b)$ :

$$
\left.\Delta \nu=\Delta v_{h}\left[\left(g(0) z / \ln \left(e^{g}(0) z+1\right)-\ln 2\right)-1\right)\right]^{k}
$$


Therefore for the filter bandwidth we have:

$\Delta v_{f}=$

$=\Delta \nu_{\text {trans. peak }},\left[\left(g(0) z / \ln \left(e^{E(0) z+1)}-\ln 2\right)-1\right]^{1 / 2}\right.$

To determine the rest of the parameters we make the following assumptions and find the respective loss factor a for Esch section of our syetems

1. Equal length distribution, that is the length of the dye amplifier is equal to the length of the filter amplifier:

$z=1_{a}$

2. Distributed losg over la (smeared):

$I_{2}=T_{\min } I_{1}=I_{1} \exp \left(-\alpha_{f} I_{a}\right)$

$\ln \left(T_{\min }\right)=-a_{f}{ }_{a}$

The filter loss factor, $\alpha_{f}$ would bes

$a_{f}=-\left[\ln \left(T_{m i n}\right)\right] / 1_{a}$

3. Smeared mirrors:

$I_{2}=R_{1} R_{2} I_{1}=$

$=I_{1} \exp \left(-2 \alpha_{m} I_{a}\right)$ 
$\delta \mathbf{E}_{\mathbf{s}} / 8 z+1 / v \delta \mathbf{E}_{\mathbf{s}} / 8 t=$

$=-a_{s} E_{s} / 2-\mu \omega_{s}{ }^{2} N / k_{s} \quad n^{*}{ }_{s} x d x-\mu \omega_{s}{ }^{2} N_{f} n^{*} f^{\prime} k_{s}-a_{f} E_{s} / 2-a_{m} E_{s} / 2$ (59-e)

where $d$ is the strength of the unsaturating amplifier and the $\alpha$ 's are the logs terms.

THE STEADY STATE LIMIT AND NORMALIZATION

The steady state golution 1s useful because it suggests a normalization. To study the filter effect by 1tgelf on the EM fleld, we set all the time derivatives equal to zero and solve for the E's response:

From eq. $(59-c)$ :

$$
\begin{aligned}
& \delta \boldsymbol{\eta}_{\mathbf{g}} / \delta t=0
\end{aligned}
$$

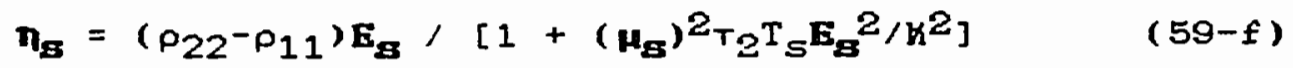

From eq. (59-d):

$$
\boldsymbol{n} \cdot \mathbf{f}=-d T_{f}\left|\mu_{\mathbf{f}}\right|^{2} \mathbf{E}_{\boldsymbol{g}} / 2 \boldsymbol{K}
$$

and from the fleld eq. (59-e): 


$$
\begin{array}{r}
\delta E_{G} / \delta z+\alpha_{g} E_{\sigma} / 2=\mu \omega_{s}{ }^{2} N_{f} d T_{f}\left|\mu_{f}\right|^{2} E_{\sigma} / 2 k_{\sigma} h= \\
=g_{f}(0) E_{\sigma} / 2
\end{array}
$$

By introducing suitable normalized forms for the dependent variables, we can write our set of equations in a more compact form. We normalize the field in the form of a parameter called A to become equal to 1 for when the steady state gain drops to $1 / 2$ its amall gignal value, ag per eq. (59-f)

$$
\begin{aligned}
& A=\left|\mu_{g}\right| / K\left(T_{2} T_{g} / 2\right)^{K} E_{g} \\
& D=\mu \omega_{s}{ }^{2} N T_{s}\left|\mu_{g}\right|^{2}\left(\rho_{22}-\rho_{11}\right) / k_{s} K a \\
& M=\mu \omega_{g}{ }^{2} T_{g} \mid \mu_{g}{ }^{2}\left(\rho_{22}+\rho_{11}\right) / k_{s} k \sigma
\end{aligned}
$$

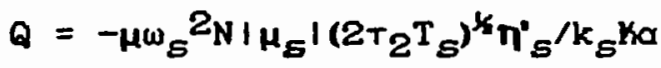

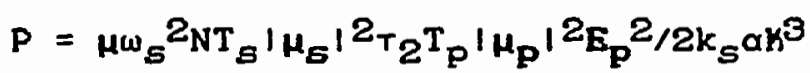

$$
\begin{aligned}
& F=-2 K\left|\mu_{s}\right| T_{2} T_{s} \eta^{\prime} f^{/ 2 T_{f} d\left|\mu_{f}\right| 2_{K}}
\end{aligned}
$$

Here, $D$ is the normalized population difference, $M$ is the normalized population sum, $Q$ is the normalized polarization, $P$ is the normalized pump rate, $F$ is the normalized filter response, and $A$ is the normalized field. With these new variables, our set of equations becomes: 
$\delta D / \delta t=$

$-1 / T_{2}\left[\left(1+T_{2} / 2 T_{1}\right) D+\left(1-T_{2} / 2 T_{2}\right) M+2 Q A x-P(T) x^{2}\right] \quad(80-a)$

$\delta M / \delta t=$

$-1 / T_{2}\left(T_{2} / 2 T_{1} D+T_{2} / 2 T_{1} M-P(T) x^{2}\right) \quad(80-b)$

$\delta Q / \delta t=-(Q-A D x) / T_{\delta} \quad(80-c)$

$\delta F / \delta t=-(F-A) / T_{f} \quad(60-d)$

$\delta A / \delta z+1 / v \delta A / \delta t=-\alpha / 2\left(A-\int Q x d x-E_{f} F / \alpha\right)$

SYNCHRONOUS PUMPING

Now we take account of the timing consideration imposed by synchronous pumping by including them in a boundary condition. Swearing the length of the dye jet $l_{a}$ in the single path length of the dye laser cavity, L. The last equation can be written:

$\delta A / 8 z+1 / v \delta A / 8 t=$

$=-a / 2\left(A-\int Q x d x-E_{f} F / a\right)\left(1_{a} / L_{d} y e\right)$

Introducing a new time coordinate:

$T=t-z / v_{s}$ 
where $v_{5}$ is the envelope speed for the steady state pulse in the dye. Imposing a steady state pulse requirement, our set of equations change from partial differential equationg to ordinary differential equations. Accordingly, the fleld equation (Bi) becomes:

$\mathrm{dA} / \mathrm{dT}=$

$=-v a / 2\left(1-v / v_{B}\right)\left(1 / L_{d y e}\right)\left(A-\int Q x d x-g_{f} l F / \Delta_{1}\right)$

where $\alpha$ is the sum of all the losses:

$$
\begin{aligned}
& a= \\
& a_{s}+a_{f}+a_{m}= \\
& =a_{s}-\ln \left(T_{m i n} / 1\right)-\ln \left(R_{1} R_{2} / 21\right)
\end{aligned}
$$

and from:

$$
\begin{array}{r}
e^{-2 a 1=} e^{-t_{r t}}{ }^{/ t_{c}}= \\
=e^{-2 L / v t_{c}}
\end{array}
$$

we obtain:

$t_{c}=L_{d y} e^{/ a l v}$

introducing: 


$$
\beta=e_{f} 1 / a l
$$

we had from eq. (58):

$$
B_{f}=\ln T_{\max } / T_{\min }
$$

Therefore the field equation becomes:

$$
\begin{aligned}
& d A / d T= \\
& -L / 2 t_{c} \Delta L\left(A-\int Q x d x-B F\right)
\end{aligned}
$$

and our set of normalized equations transforms into:

$$
\begin{aligned}
& d D / \delta t= \\
& -1 / T_{2}\left[\left(1+T_{2} / 2 T_{1}\right) D+\left(1-T_{2} / 2 T_{2}\right) M+2 Q A x-P(T) x^{2}\right] \\
& d M / \delta t= \\
& -1 / T_{2}\left(T_{2} / 2 T_{1} D+T_{2} / 2 T_{1} M-P(T) x^{2}\right) \\
& d Q / \delta t=-(Q-A D)^{\prime} \\
& (80-b)^{\prime} \\
& d F / \delta t=-(F-A) / T_{f}(60-c)^{\prime} \\
& d A / \delta z=-L / 2 t_{C} \Delta L\left(A-\int Q x d x-B F\right) \\
& \text { (60-d)' }
\end{aligned}
$$


In our modelling, we have used a Gauggian pump function in the forms

$$
\begin{aligned}
P(T)= & P_{0} 2 / \Delta T[(\ln 2) / \pi]^{1 / 2} \exp \left[-(2 T / \Delta T)^{2} \ln 2\right] \\
= & P_{0} f(T)
\end{aligned}
$$

where $f(T)$ is normalized so that:

$$
\int f(T) d T=1
$$

Defining the threshold parameter $r$ as:

$$
r=P / P_{t h}=1 / A\left(\int Q x d x-\beta\right)
$$

we can determine $P_{0}$ by setting $2 Q A x$ equal to zero in eq. (80-a) and solve for the value of $r$ in eq. (87) from the get of equations $80(a-d)$ and $(63)$.

\section{SUMMARY}

In this chapter we have developed, a theoretical set of equations that describe a synchronously pumped mode-locked dye lager system with a bandwidth limiting element. The inclusion of the bandwidth tuning effects which have added a new equation, eq. (80-d), and the coupling terms in eq. (B3). In the next chapter we will show the theoretical solutions and the 
parameters that we used, together with the degcription of the experimental regults that verify our theory.

Figure 3 shows a sample theoretical solution plot of Pump function which represents $P$ in the equations, Gain which represents $D$ (population difference), Intensity of the dye output pulge which represents $A^{2}$, and the auto-correlation of the output pulse intensity.

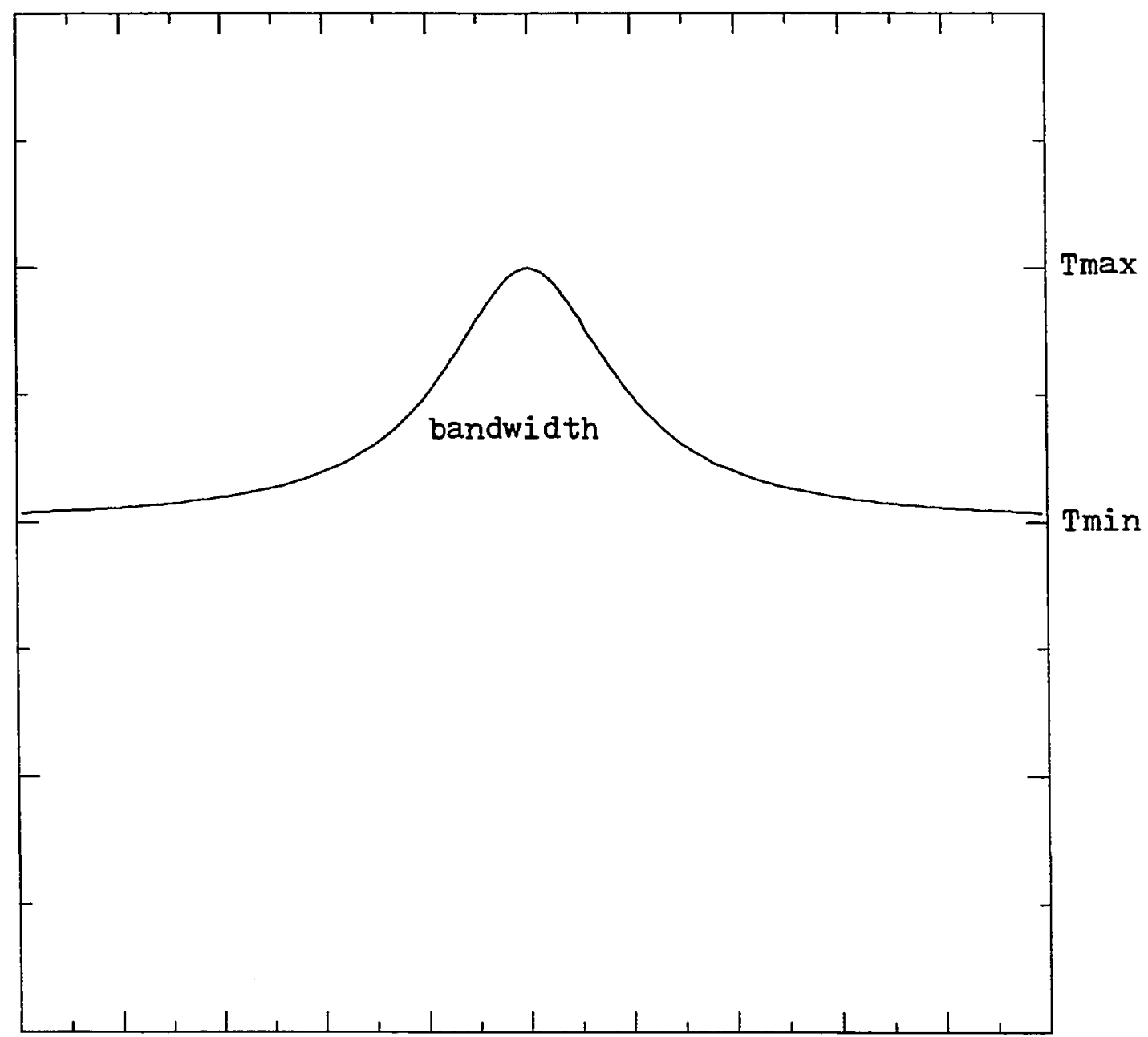

Figure 2. Lorentzian line shape. 


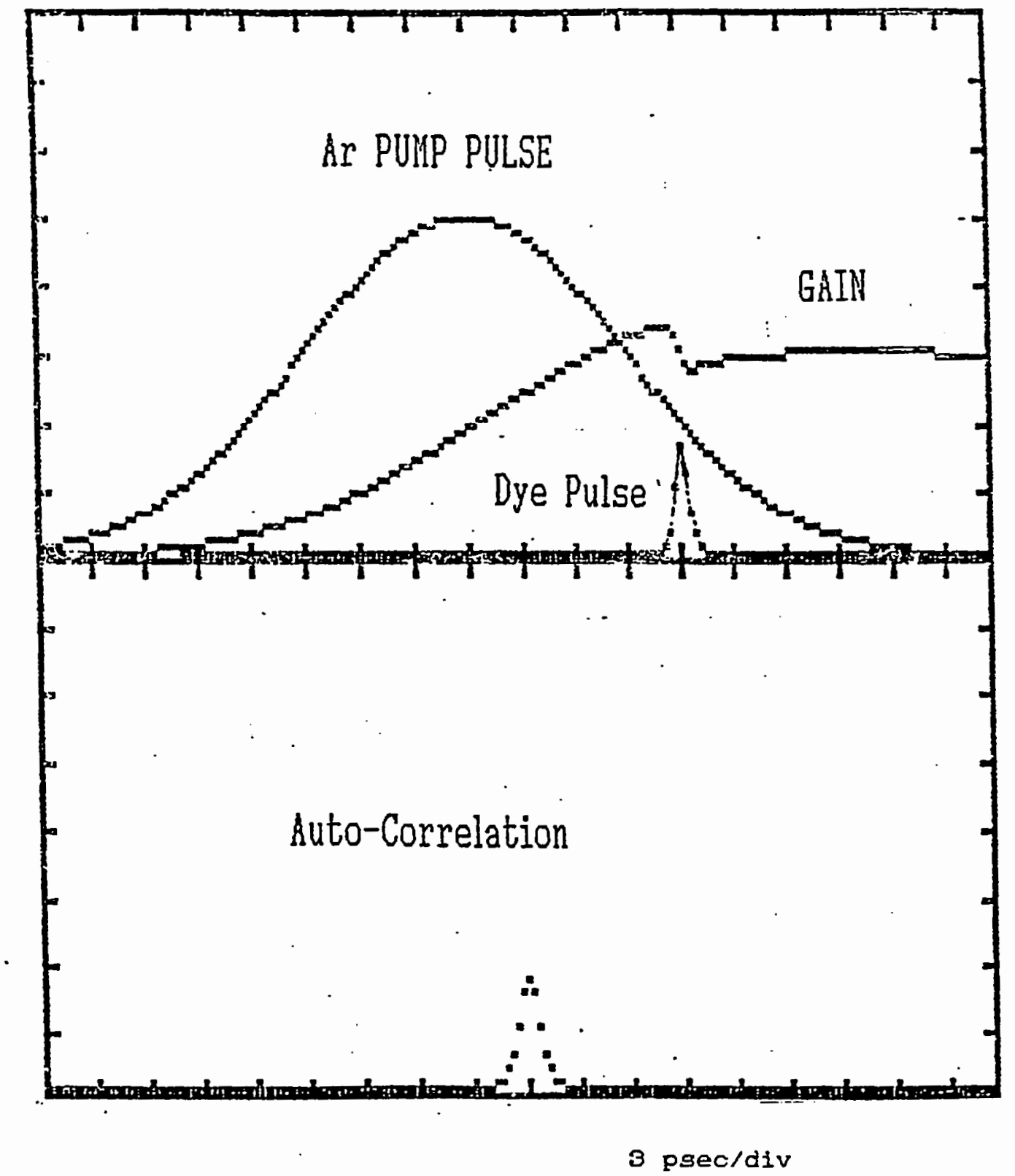

Figure 0. Sample theoretical plot. 
CHAPTER III

\section{EXPERIMENT}

In order to verlfy the theoretical model, we degigned a series of experiments. In these experiments, an acoustooptically mode-locked argon lon lager (Spectra-Phyglcg Series 2000) was used to synchronously pump a rhodamine $6 G$ dye laser (Spectra-Physics model 375B). To study the tuning effects, we have used two and three plate birefringent filters and a tuning wedge. The autocorrelated pulses have been measured as a function of the length detuning using a Spectra-Physics Model 409 Scanning Autocorrelator.

Figure 4 shows the general schematic of a synchronously pumped mode-locked system. It involves two lasers. The firgt laser, used to pump the amplifier medium of the second laser, is a mode-locked laser generating a continuous train of pulses at repetition rate $c / 2 L_{1}$, where $L_{1}$ is the optical length of the first laser's regonator. The second laser, with a resonator optical length $L_{2}$, uses an amplifier medium characterized by a recovery time shorter than the time Interval $2 \mathrm{~L}_{1} / c$ extsting between two consecutive pulses of the pump laser. Under the pump excitation, the gain of the second laser is then modulated at the frequency $c / 2 L_{1}$. The 
second laser will operate in a mode-locking regime, if its intermode spacing, $c / 2 L_{2}$, is adfusted to the frequency of the gain modulation, c/2L $L_{1}$. This condition can be achieved by forcing the length of the two laser cavities to be equal.

\section{ARGON LASER PUMP}

The dye laser is pumped by the argon laser described in this section. The Spectra Physics 2020 Argon Ion laser 1s a high current plasma device that emits radiation at $514.5 \mathrm{~nm}$. It is actively mode-locked and produces an $80 \mathrm{MHz}$ train of 100 psec. pulses.

In order to have a traveling packet of light circulating inside the cavity of the argon laser, we need to force the longitudinal cavity modes of the laser to maintain a fixed relationship with each other. This process is called mode-locking and it is produced by modulating the cavity losses at a frequency equal to the frequency difference between adjacent longitudinal modes. The mode separation frequency $\Delta \nu$ is:

$\Delta \nu=c / 2 L$

where $c$ is the speed of $11 \mathrm{ght}, \nu$ is the frequency and $L$ is the laser cavity optical length. We produce the loss modulation by an acousto-optic modulator. 
The index of refraction of a material changes periodically if a sound wave is passed through 1t. This is cauged becauge of the change in dengity of the materisl when a sound wave is passed ingide 1t. In our setup the sound waves are produced by a plezoelectric transducer which convertg electrical aignalg into acoustic energy. Once the sound passeg through the material, the index of refraction will change periodically. Now if a light beam also passes through the same material, it will be partially diffracted off this perlodic refractive grating. The laser inftially has a given amount of energy traveling within its tube. On each pass through the mode-locker, that portion of the energy arriving when the modulation function is at a minimum wlll be trangmitted through the mode-locker. Energy arriving before or after that time will be attenuated. After several passes, the energy in the laser is in the form of a pulse which is in synchronization with the mode-locker. We measured Ar pulsewldth of 80 ps with the average power of $450 \mathrm{mw}$ and modulating frequency of $40.9120 \mathrm{MHz}$.

THE SYNCHRONOUSLY PUMPED DYE LASER

We used the Spectra-Physics Model $375 \mathrm{~B}$ dye laser system for our experiments. It consists of a three mirror regonator (Figure 5) [31], 1.e. two spherical reflecting confocal mirrors and $a$ third exit mirror; and a fet of the 
organic dye in a solvent with high viscosity (ethylene glycol). The pump radiation is focused in the dye jet by a short-focus lens: synchronous operation is achieved by plane-parallel displacement of the exit mirror to match the lengths of the dye and the pump lasers.

The organic dye used in our setup was Rhodamine $6 \mathrm{G}$ which its energy levels was shown in Figure 1 and discussed in chapter II.

\section{EFFECTS OF LENGTH DETUNING}

The round trip time of the dye pulse within the dye optical resonator is fixed by the repetition rate of the pump pulse. The resulting length matching between the two laser cavities is then rather critical. To some degree, pulse reshaping in the amplifier medium can automatically compensate for small induced changes in the round trip propagation time, thus length mismatches on the order of 100 microns can be tolerated without destroying stable operation in the system.

This reshaping process can be qualitatively explained by considering the saturation of the gain induced by the dye pulse in the amplifier medium. Before the pulse arrival, the gain is unsaturated. Thus, the leading edge of the pulse experiences a larger gain than the trailing edge. This preferential amplification of the leading edge of the 
pulse produces a new pulse that is slightly advanced, so the transit time of the pulse across the amplifier medium is shortened. The advance experienced by the dye pulse in the amplifier medium is directly related to the value of the ungaturated gain at the crosging time and occurs regardlegs of the lager cavity length permitting a stable operstion. In particular, this reshaping effect explaing the slight positive length migmatch $\left(L_{2}=L_{1}+e\right)$ that characterizes the test modelocking operation in synchronously pumped systems.

If we shorten the dye laser cavity slightly from its optimum length, the dye pulse clrculating within the optical resonator will arrive at the amplifier medium earlier than would be optimum. Since the value of the unsaturated gain Is proportional to the Integral of the pump pulse over time, the leading edge of the dye pulse experienceg lower gain than in the optimum position. The corresponding decrease in the efficiency of the reshaping process produces an increase In the effective transit time, which can then permit continued stable modelocking of the dye amplifier.

If we lengthen the laser cavity, the dye pulse is retarded by the longer cavity and arrives at the dye later than optimum. But it geeg higher ungaturated gain than optimum, so 1ts transit time through the amplifier medium is reduced accordingly. Thus slight changss of the dye laser cavity during gtable operation induce a readjustment of the 
dye pulse shape. Lengthening the cavity retards the dye pulse, moving it further out in the gain curve past the point where the gain curve and logg line intergect. Becauge of the larger value of the ungaturated gain, the pesk Intensity of the pulse increases. At the same time, the pulse broadens to match the cavity round trip time with the period of the pump pulse. After reaching a maximum, the peak intensity of the pulse decreases for large cavity detuning (greater than $50 \mathrm{\mu m}$ ) because of the logs of unsaturated gain by spontaneous emission. Increasing the cavity length ultimately precludes complete modelocking; the characteristic "Prussian helmet" shape of the autocorrelation traces appears in the dye pulge.

If the laser cavity is shortened from the optimum position, the dye pulse moves closer to the point where the gain crosses the loss line. The peak intensity of the pulse decreases because of the lower net gain experienced, and the pulse duration glightly decreases to fulfill the pump laser timing requirements. If the gain depletion induced by the dye pulse occurs early enough, the amplifier medium (which continuely integrates the pump pulse) can recover enough gain to overcome the cavity losses. This gain recovery allows the formation of a second pulse in the laser cavity, delayed by several tens of picoseconds from the main pulse. Further length reduction decreases the delay between the two pulses and can even lead to the generation of a third or 
fourth pulge in the laser cavity. If the lager cavity is shortened until the dye pulse arrives before the gain curve crosses the loss line, the pulse is quenched. Lasing continues, however, in the form of a broadband pulse whose parameters are determined by the pump pulse and the saturation characteristics of the gain medium. The mechanism of formation of short pulses is described in Appendix A. 


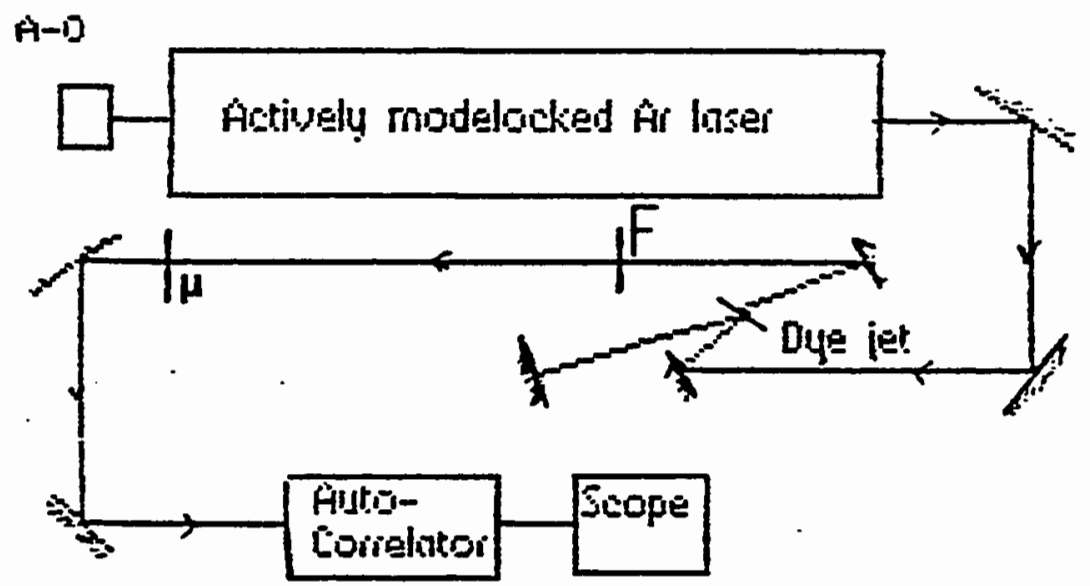

Flgure 4. Our sync. pumped modelocked dye laser 


\section{BANDWIDTH LIMITING TUNING ELEMENTS}

To 1solate a wavelength of interest we uged a tuning wedge and two different birefringent fliters. The description of these tuning elements are as follows.

\section{Tuning Wedge}

A tuning wedge consists of a fuged-gllica substrate and two mirror costings that are sepsrated by a thickness gradient as in Figure $e$ [31]. Silding the wedge scross the beam path changes the digtance that light travels between the mirrored surfaces and, with 1t, the transmission frequency. By moving the wedge in a direction perpendicular to the cavity axis, the peak transmission of the filter is tuned to a different wavelength. The reduced loss at the new wavelength admits stimulated emisgion there. A tuning wedge reduces the dye laser output linewidth to about $240 \mathrm{GHz}$. We meagured the bandwidth of the tuning wedge to be $25 \mathrm{THz}$ ag shown in Figure 10.

\section{Birefringent Filter (Lyot) $[27,28]$}

A birefringent filter conglsts of several quartz waveplates of different thicknesses (Figure 7) [32]. These plateg are placed in the laser cavity st the Brewster angle such that the vertically polarized light in the cavity 
experiences no loss by reflection at the plate surfaces. The no-loss condition permits amplification of light in the cavity with a high degree of linear polarization. The crystal axes of the quartz are oriented such that the plate behaves as a full waveplate for vertically polarized light if $\lambda_{0}$, the wavelength in a vacuum, satisfles the relation:

$$
d\left(n_{\text {slow }}-n_{\text {fast }}\right)=m \lambda_{0}
$$

where $d$ is the thicknegs of the plate and $m$ is an integer. For other wavelengths, transmission of the vertically polarized light through the plate results in ellptical polarization. After reflection at an end mirror, this elliptically polarized light experiences loss by reflection at the next encounter with the waveplate surface. This loss prevents lasing at wavelengths that differ much from those sat1sfying the fullwave condition. Tuning the laser is accomplished by rotating the plates, which are mounted on a common stage, about the normal to the plate surfaces. Because the plates are inclined to the optic axis, the rotation effectively changes the slow axis refractive index from nslow to n'slow' therefore changing the preferred wavelength to

$$
\lambda_{0}^{\prime}=d\left(n^{\prime} \text { slow }-n_{\text {fast }}\right) / m
$$

Each tuning element has its advantages and disadvantages. 
The wedge can be used over a broad wavelength range. Compared to the birefringent filter, it has high optical loss and low output power since the birefingent filter is Inserted at Brewster's angle, 1tg logs is very low. However the plates need to be allgned for optimum performance within a Iimited wavelength range. 


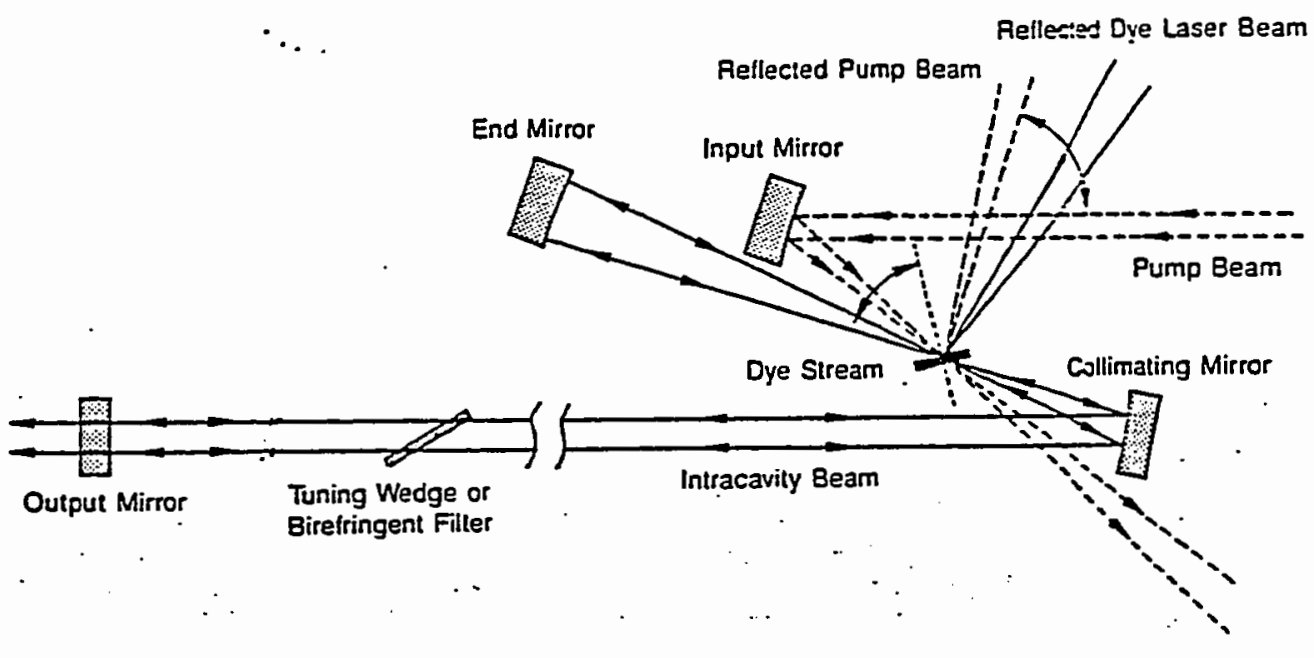

Flgure 5. Dye laser closeup [31]. 


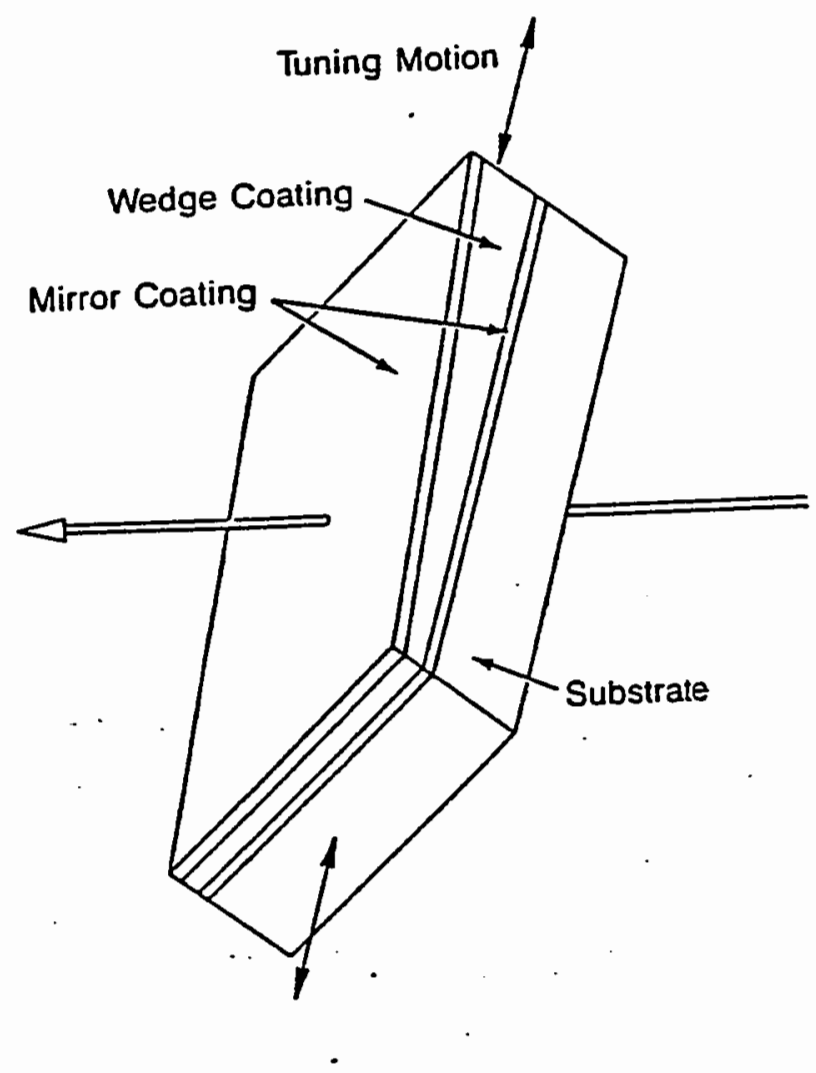

Figure 8. Tuning wedge [31]. 


\section{BANDWIDTH MEASUREMENT METHODS}

1. To determine the bandwidth and transmisaion of these filters we conducted experiments and measured the transmiasion vo wavelength for every filter. To measure the bandwidth of the tuning wedge, we uged the cary 14 Spectraphotometer. It conslats of a white light source and a grating that would allow the white light to be differentlated into different wavelengths and a recorder that plots the transmission versus wavelength.

2. For the two birefringent filters we shined different wavelengths of light through them and measured the Input and output power and therefore the transmisalon versus wavelength. We measured the wavelengths using a Jarrell-Ash Monospec/5o monochromator. The transmission ve wavelength curves for each of the fllters are shown in Figures $8,9,10$. 


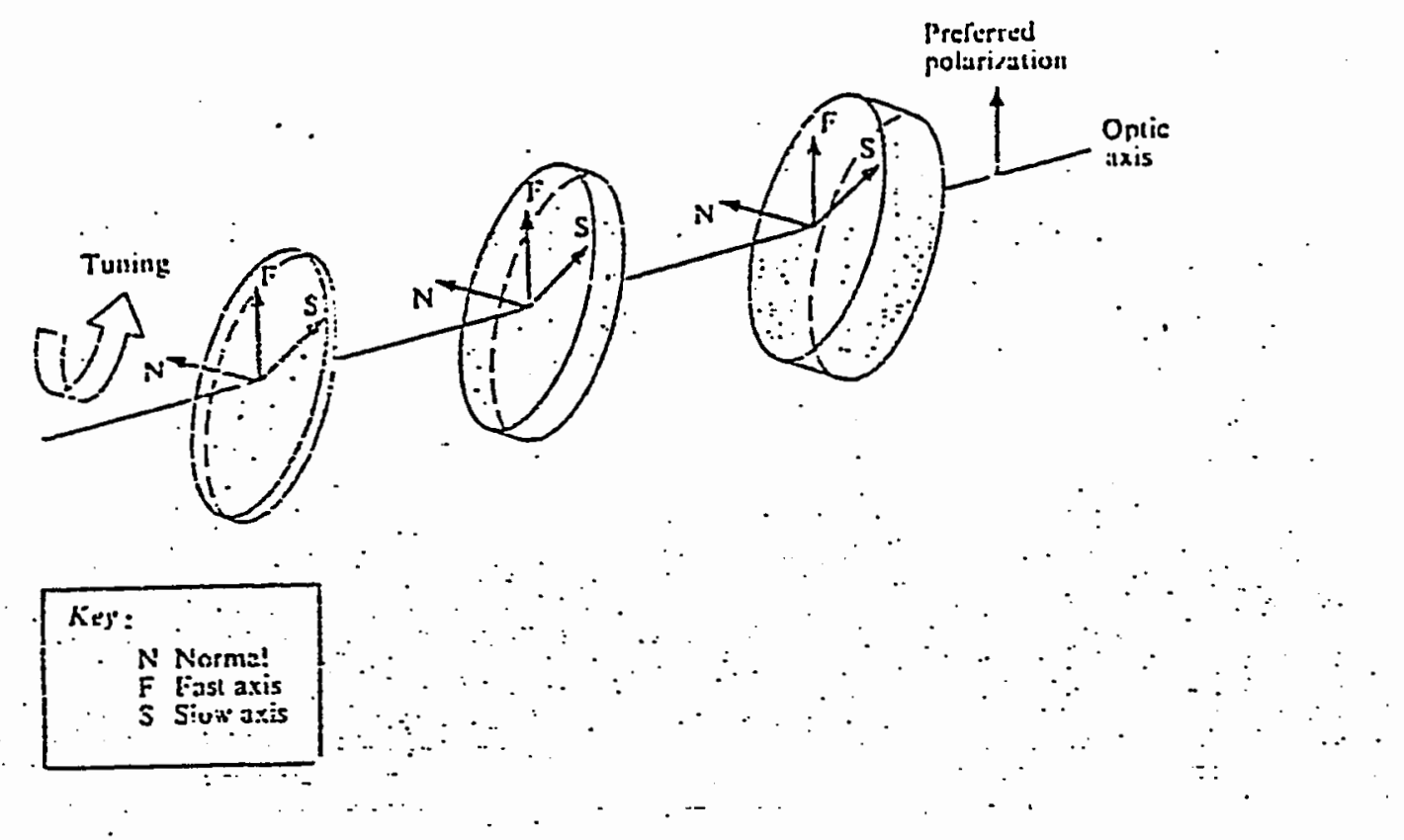

Figure 7. Birefringent filter. 


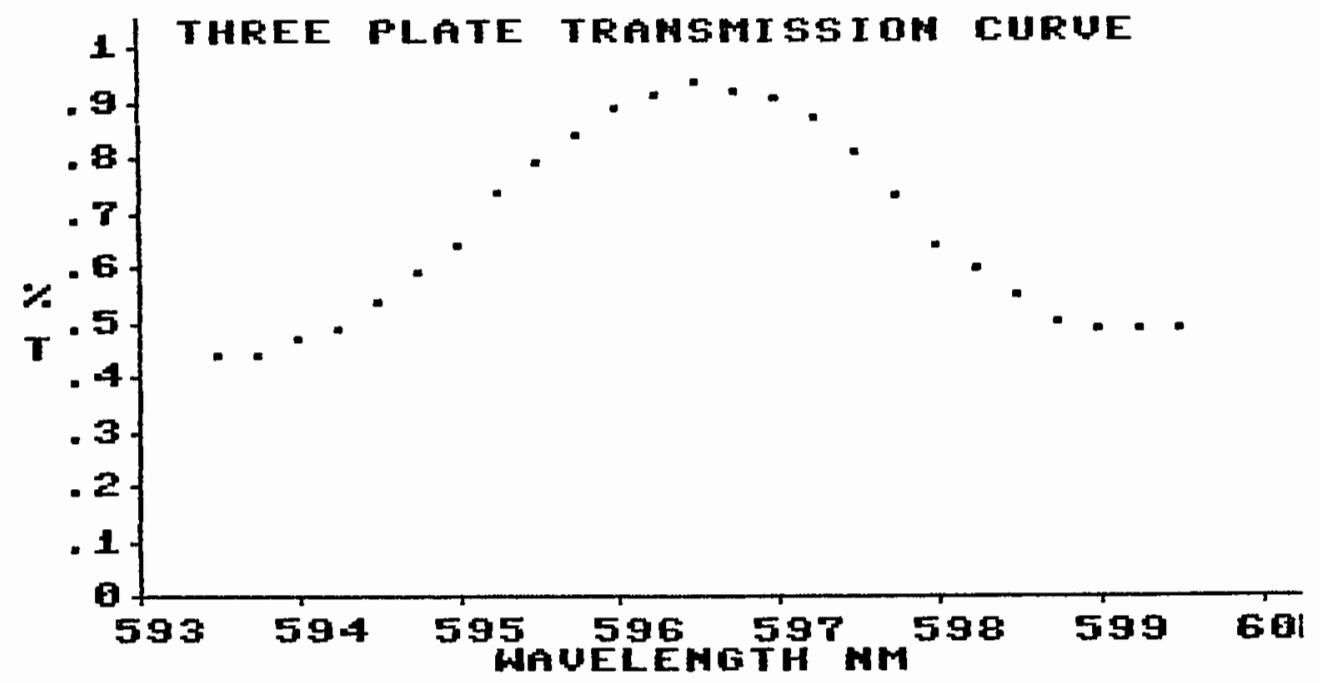

Figure 8. Three plate trangmission curve. 


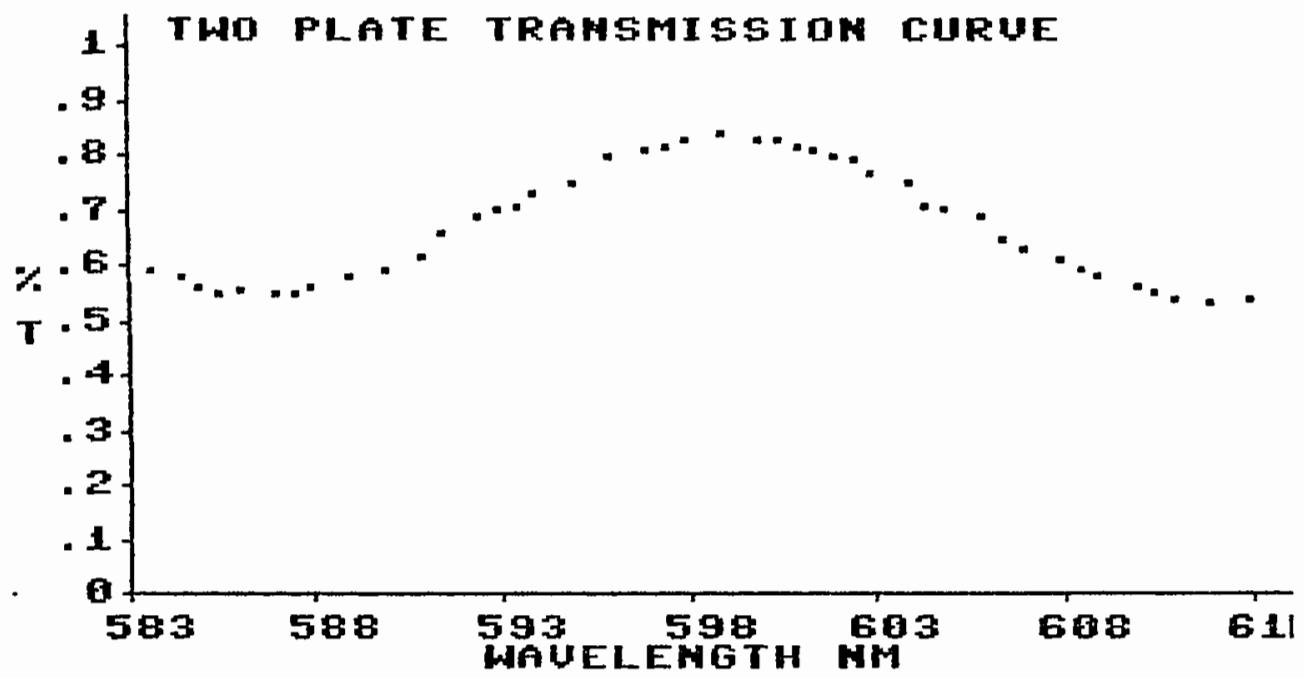

Figure 9. Two plate transmission curve.

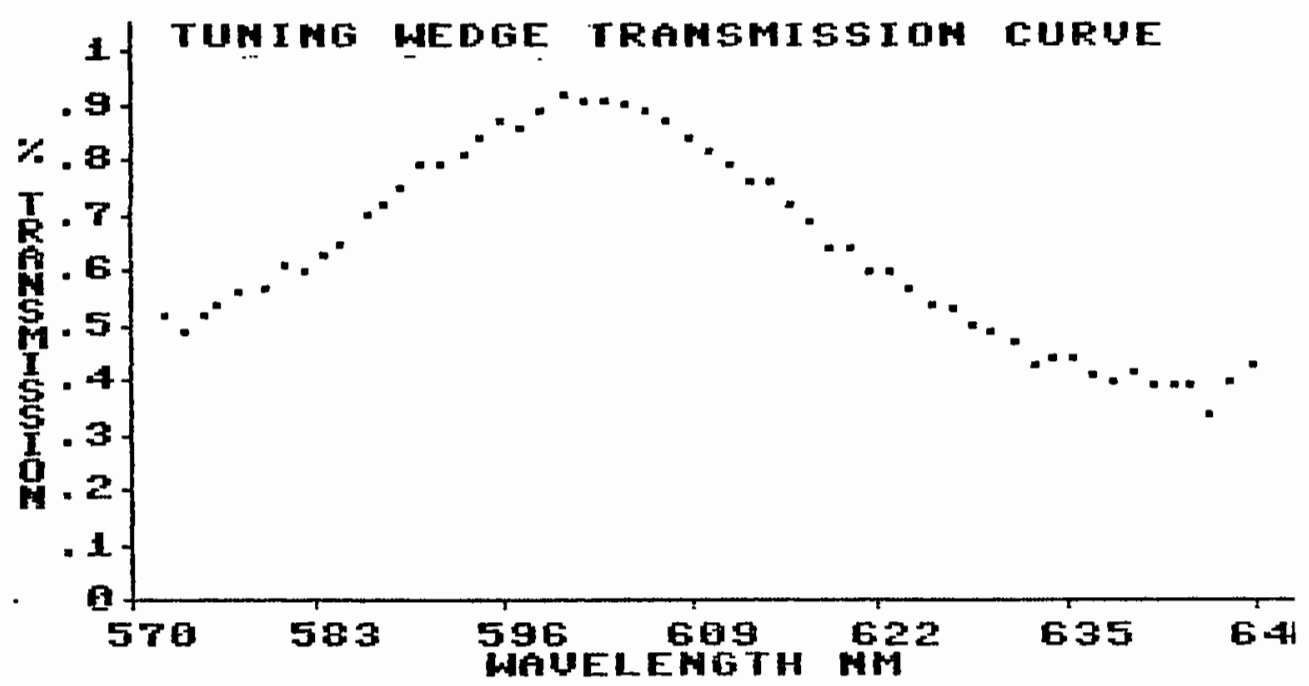

Eigure 10. Tuning wedge transmission curve. 


\section{CHAPTER IV}

\section{RESULTS AND CONCLUSIONS}

Figureg $(11,12,13,14)$ ahow the experimental autocorrelations of the output pulae shapes and the theoretical intenalty autocorrelationg regults of our model for different filter bandwidth and length detunings. The experimental data are plctureg taken from the auto-correlations of the dye lager output on a Tektronle model 7813 oscllloscope. The theoretical graphs are computer plots of the eolutiong of the equationg.

Figures $(15,18,17)$ the theoretical and experimental regults are plotted againgt each other. The solld lines are the theoretical regults and the experimental data points are shown by error barg. The error barg represent the range of accuracy of our data which 18 about $\% 10$ to $\% 15$. We notice that, the shortegt pulses are produced by the widest bandwidth, as predicted by the theory. Another observation is that the pulge widths increage with length detunings.

Table I containg the parameterg for our theoretical and experimental data. 
TABLE I

PARAMETERS

3 plate fllter 2 plate filter tuning wedge

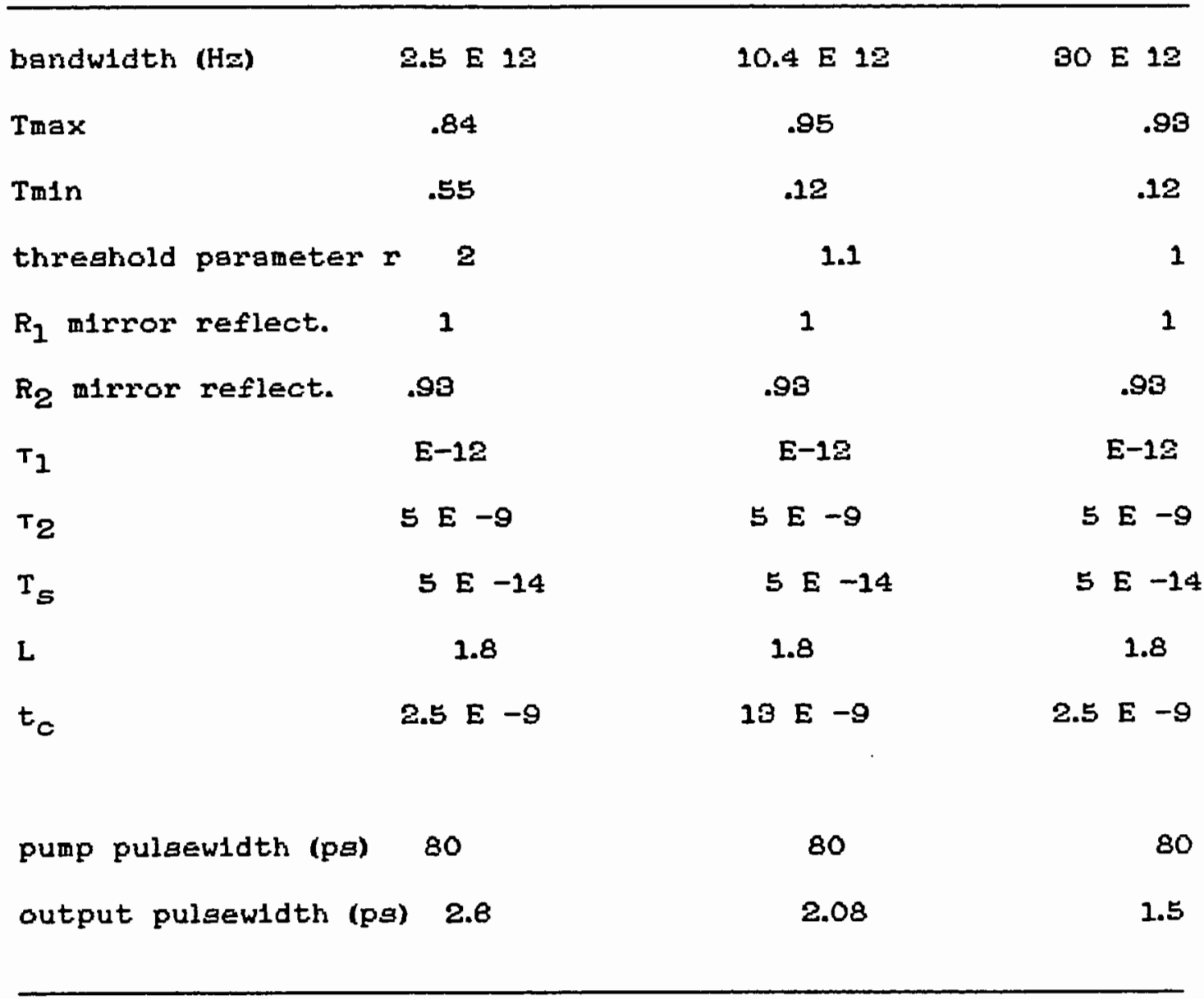



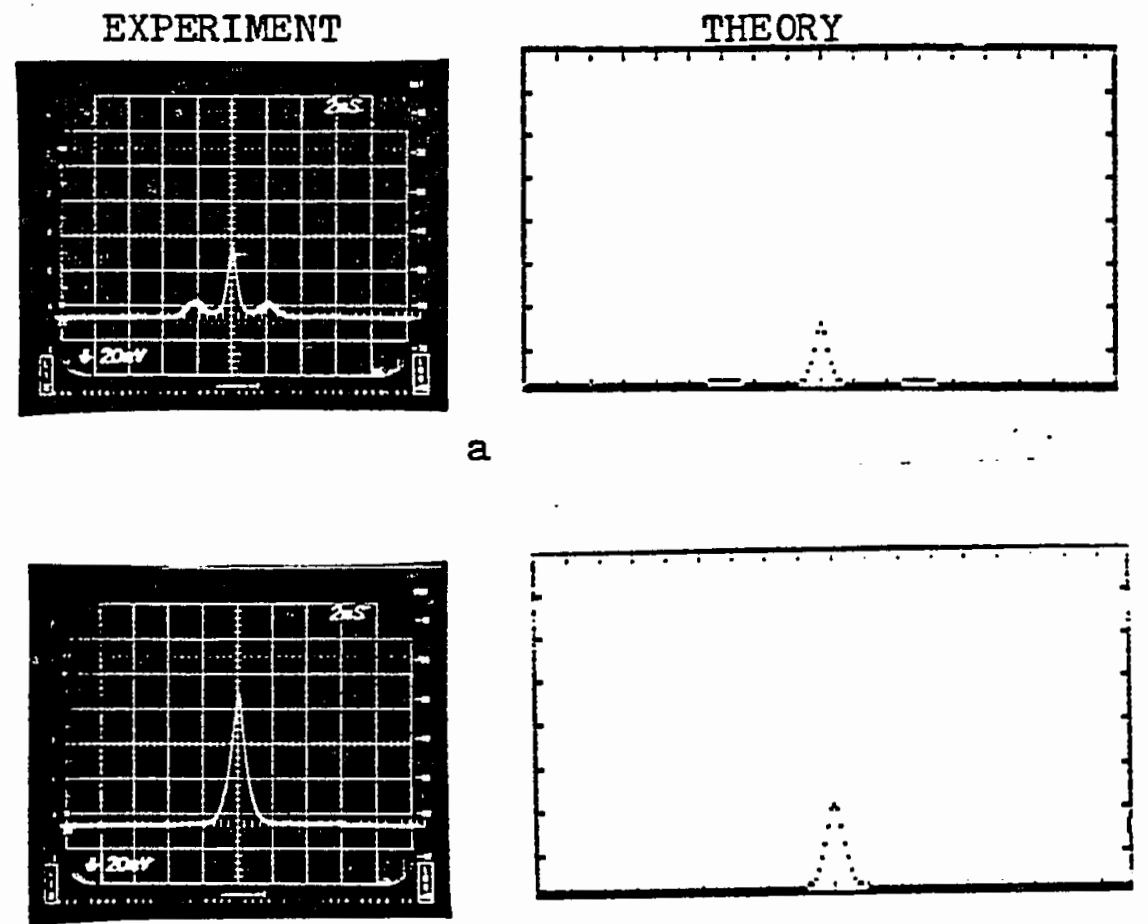

b

TINE

5 psec/div

$3 \mathrm{psec} / \mathrm{div}$

Figure 11. Autocorrelation of the output pulse for the 3 plate filter with a) $5 \mu$ and b) $18 \mu$ length detuning. 

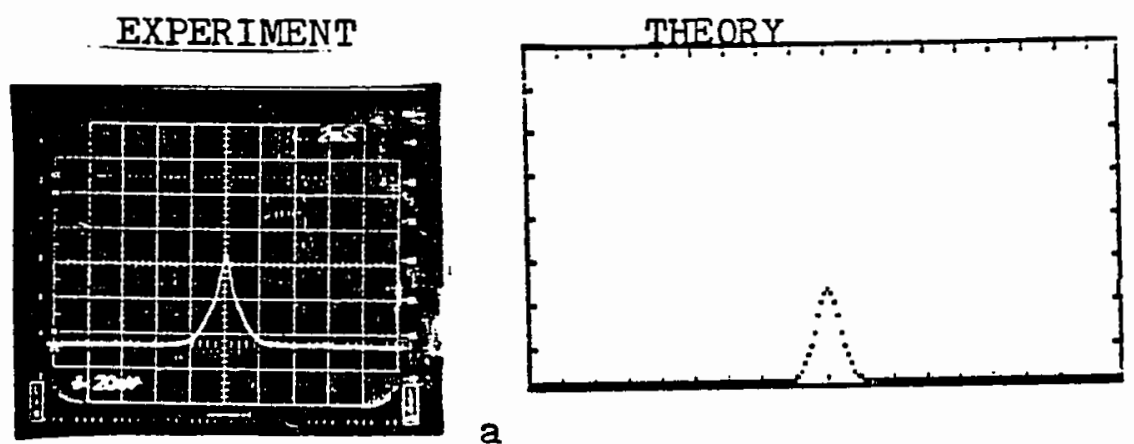

a
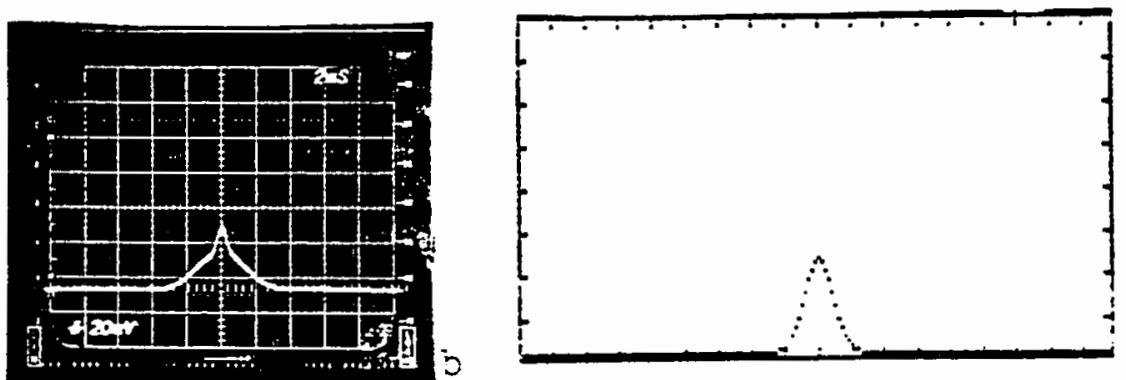

5 psec/div

3 psec/div

Figure 12. Autocorrelation of the output pulse for the 3 plate filter with a) $48 \mu$ and b) $71 \mu$ length detuning. 


\section{EXPER IMENT}
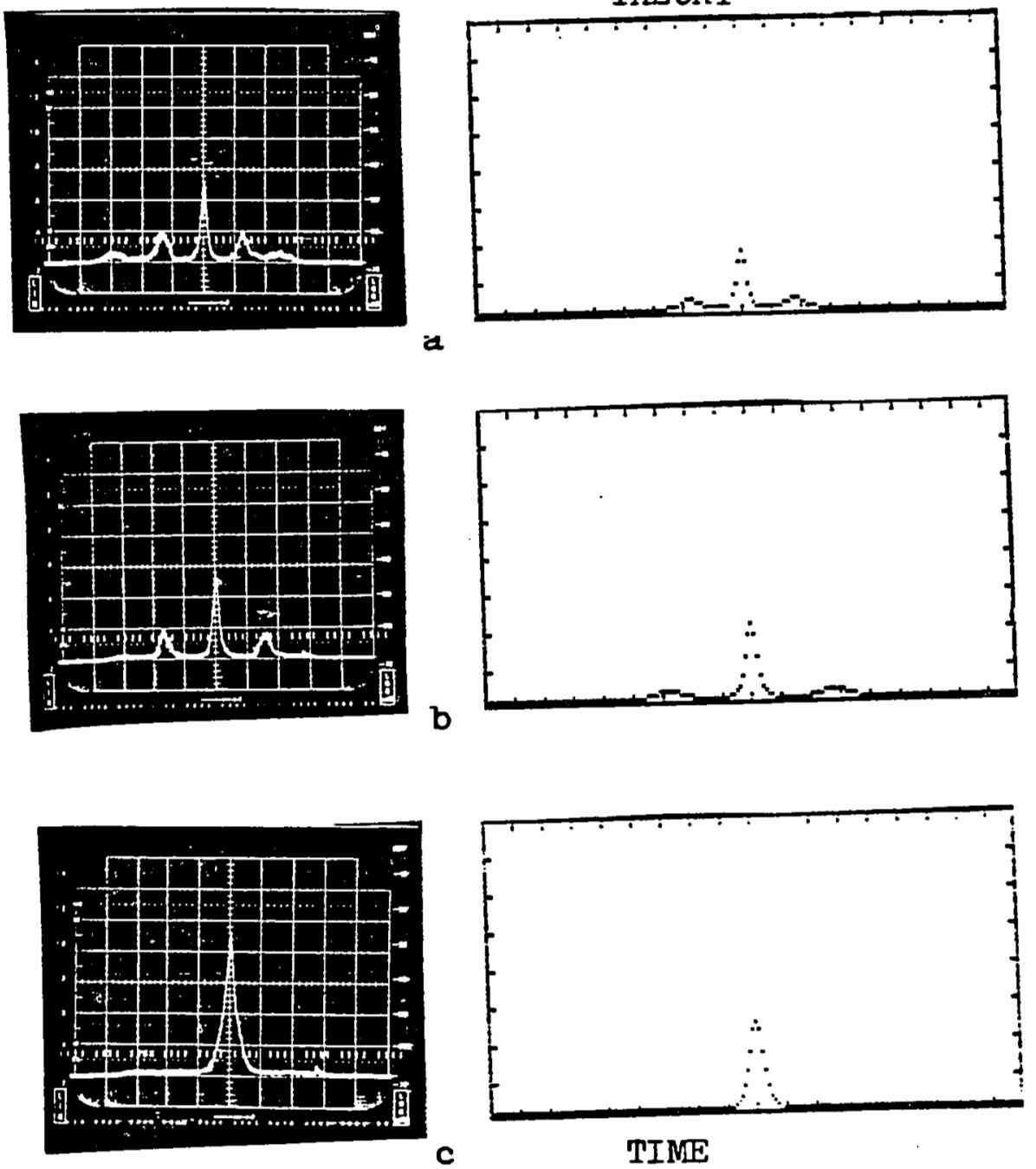

5 psec/div a

b

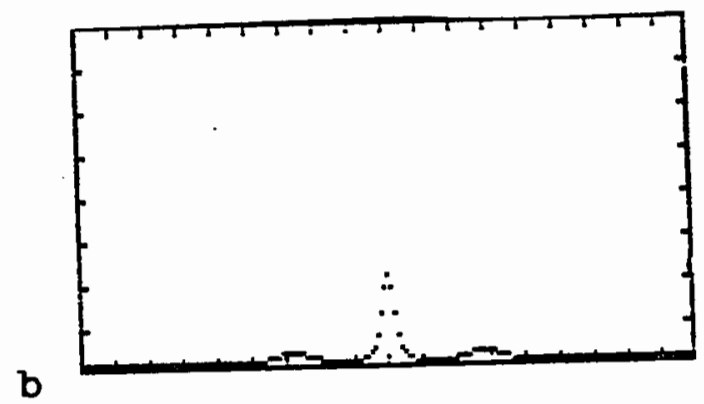

THEORY

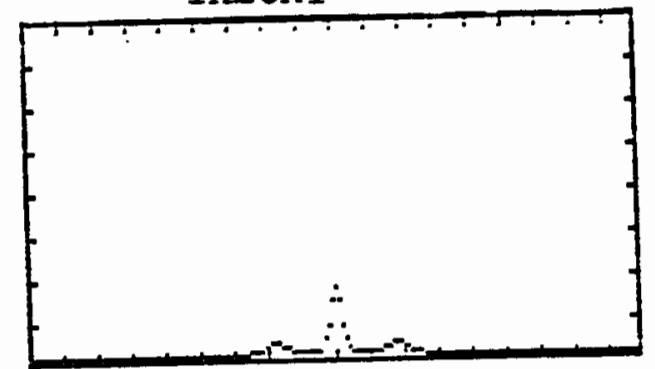

a

TIME

Figure 13. Autocorrelation of the output pulse for the 2 plate filter with a) $3 \mu, b){ }_{\mu} \mu$ and c) $11 \mu$ length 
EXPERIMENT
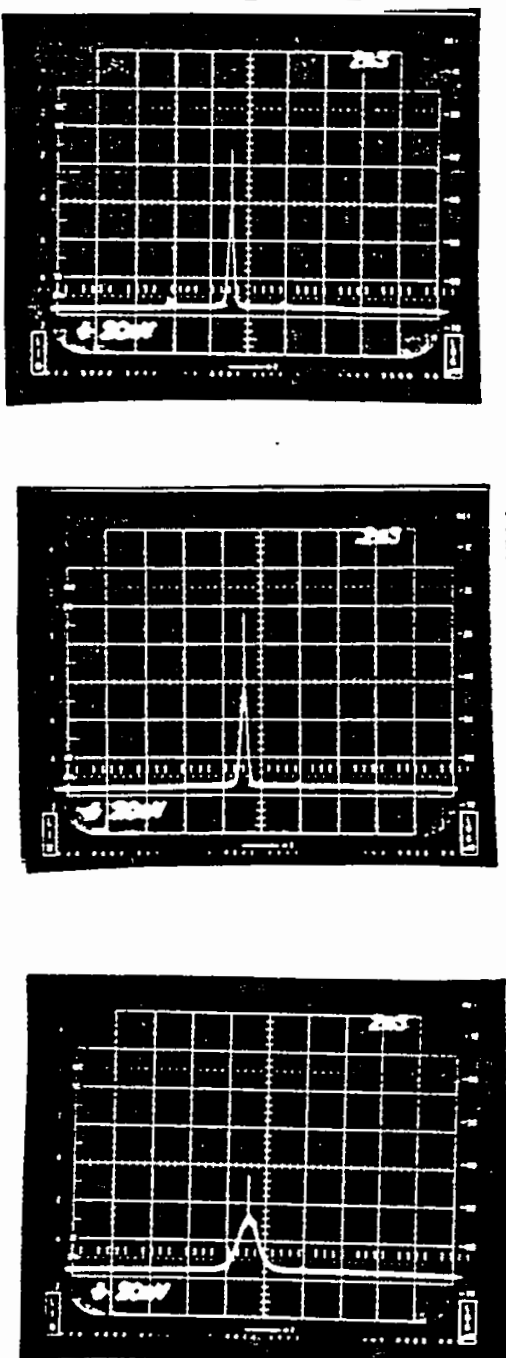

5 psec/div b

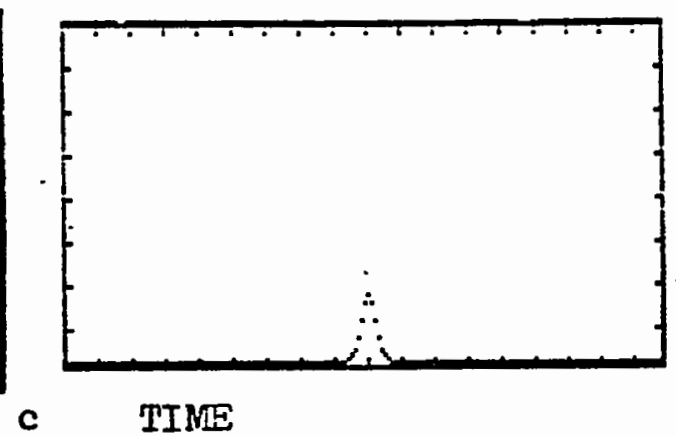

THEORY

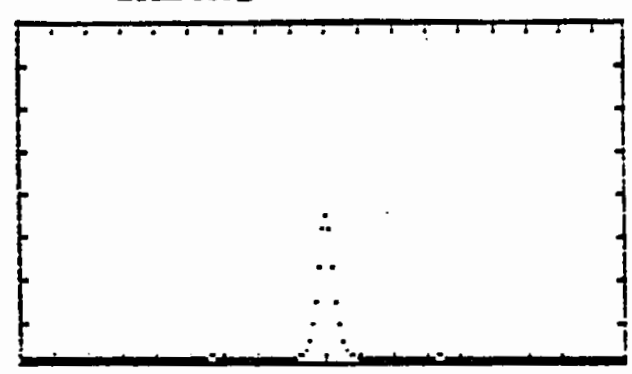

a

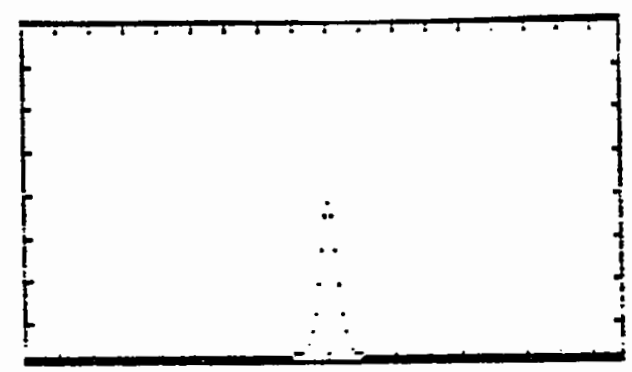

3 psec/div

Figure 14. Autocorrelation of the output pulse for the tuning wedge with a) $2 \mu$, b) $6 \mu$ and c)2ik length detuning. 


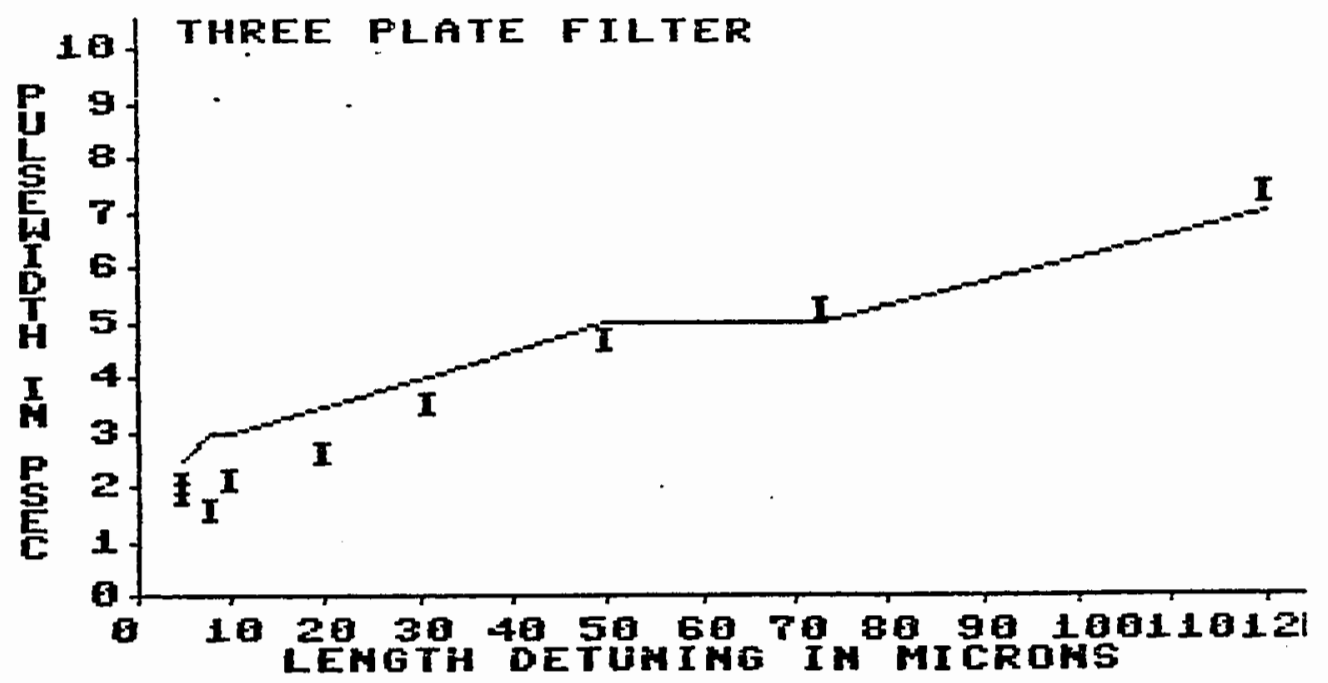

F1gure 15. Theoretical line and experimental bare for the 3 plate fllter. 


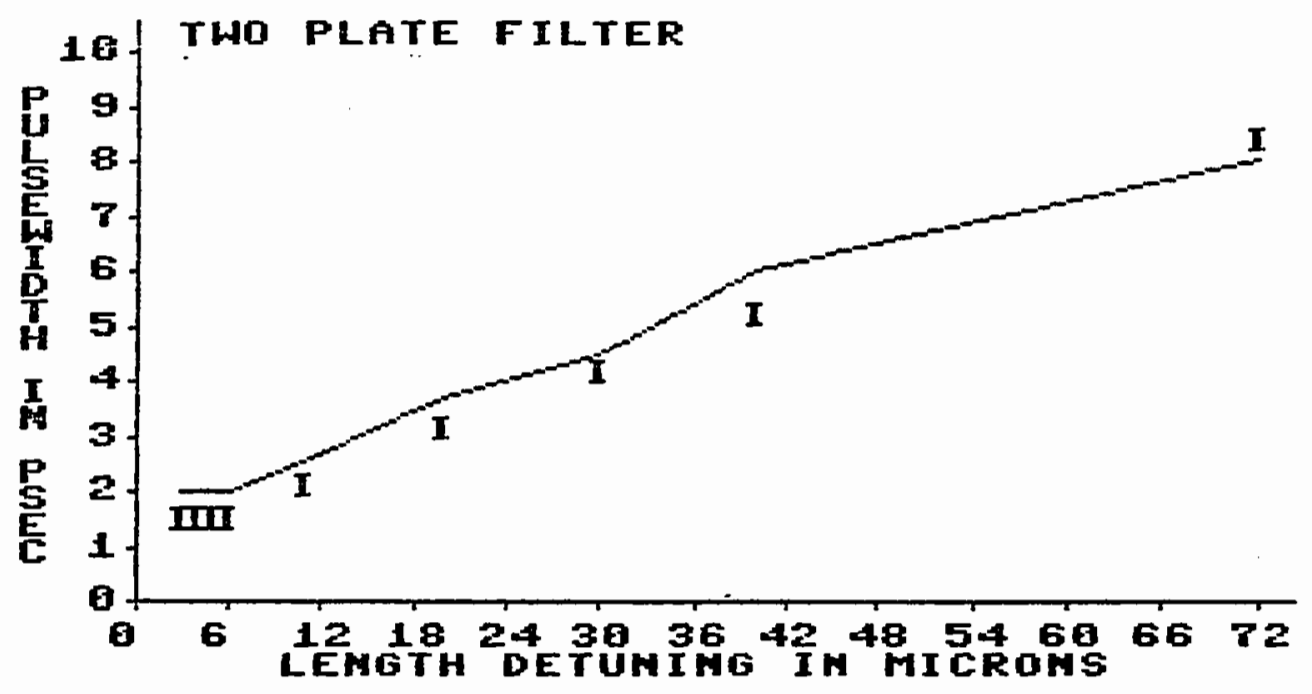

Figure 18. Theoretical line and experimental bars for the 2 plate filter. 


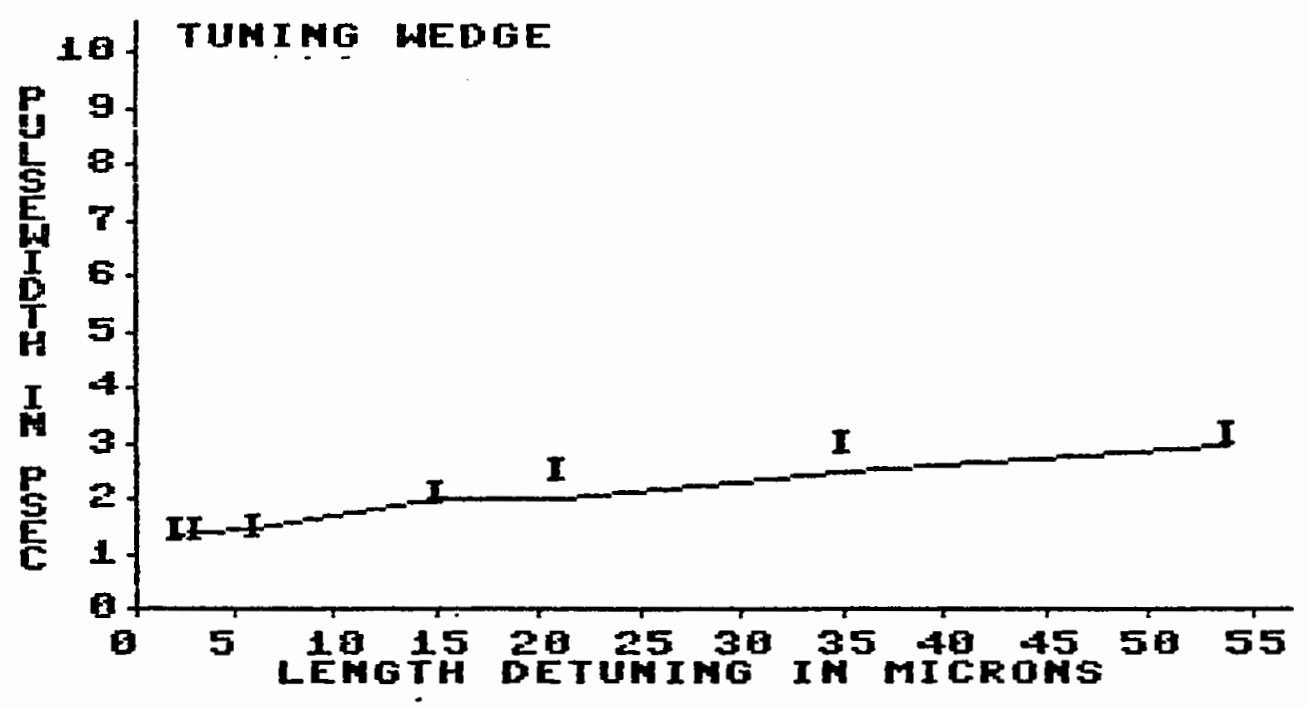

Figure 17. Theoretical line and experimental bare for the tuning wedge. 
CONCLUSIONS

In thig otudy we have experimentally verifled a semiclasalcal model for aynchronougly pumped mode-locked dye lasers producling tunable plcosecond pulses. An 1mproved description of the bandwidth limiting tuning fllter has been introduced within a semi-classical model. The new set of equations has been solved numerically ualng the best avallable values for the various parameters, and autocorrelations have been computed for a range of different length detuning and bandwidth limiting elements. The experimental pulge ghapes agree clogely with the theoretical solutions for all valueg of detuning and filter bandwidth. In the next chapter, through a gengitivity analysis, it 1g shown how this model can be used for englneering deglgn purposeg to gelect values for the transmiasion and the bandwidth of the fliter to obtain optimum pulse characteristics. 


\title{
CHAPTER $V$
}

\author{
FURTHER NUMERICAL STUDIES
}

\section{SENSITIVITY ANALYSIS (ENGINEERING DESIGN)}

Now that the theory has been verlfled experimentally, we can gtudy how the theory behaves numerlcally on a computer. We have two different parameters for our model of the filter:

1. Bandwidth of the filter $T_{f}$.

2. The ratio $[\ln (\operatorname{Tmax} / \operatorname{Tm} 1 n)] /$ losses $=\beta$

We now study the behavior of the theoretical pulse characteriatica as a function of the two parameters agsoclated with the filter. Among the various factors there are the two most pertinent:

1. The Peak Intensity Ipeak-

2. The Pulsewidth Tpulge*

We study these two parameters as a function of $\beta$ and $T_{f}$ as shown in flgures 18-21. In cage of $\beta$, we have kept the oum of losges ( $\alpha$ ) congtant, therefore the only parameter changing in $\beta$ is $T_{\text {max }}$ For englneering degign, these curves can be used to obtaln a fliter element with desired parameters. From the plots we can observe that: 
a. Low $B$ values produce high peak intenglties and ghorter pulseg. This meang lower $T_{\text {max }}$ leads to higher peak intengitieg and shorter pulses.

b. Large $T_{f}$ produce shorter pulses.

c. Peak intengitieg are ingengitive to $T_{f}$.

\section{SUGGESTIONS FOR FURTHER STUDIES}

Further work can be guggested in cages which are not consldered in this study. These include the following one can study speclalized fllter shapes instead of our Lorentzian. Also filter detunings from the line center of the dye can be studied. 


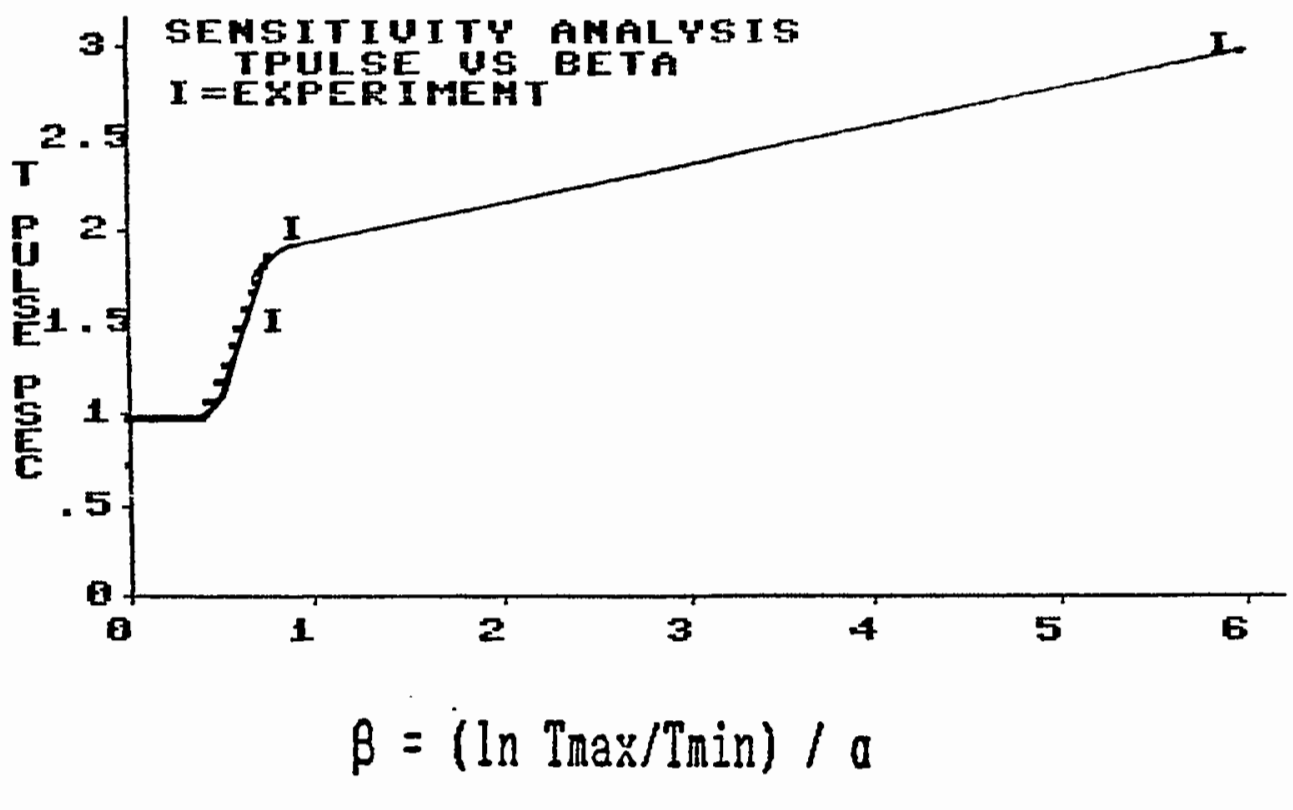

Figure 18. Pulsewidth vs $\beta$. 


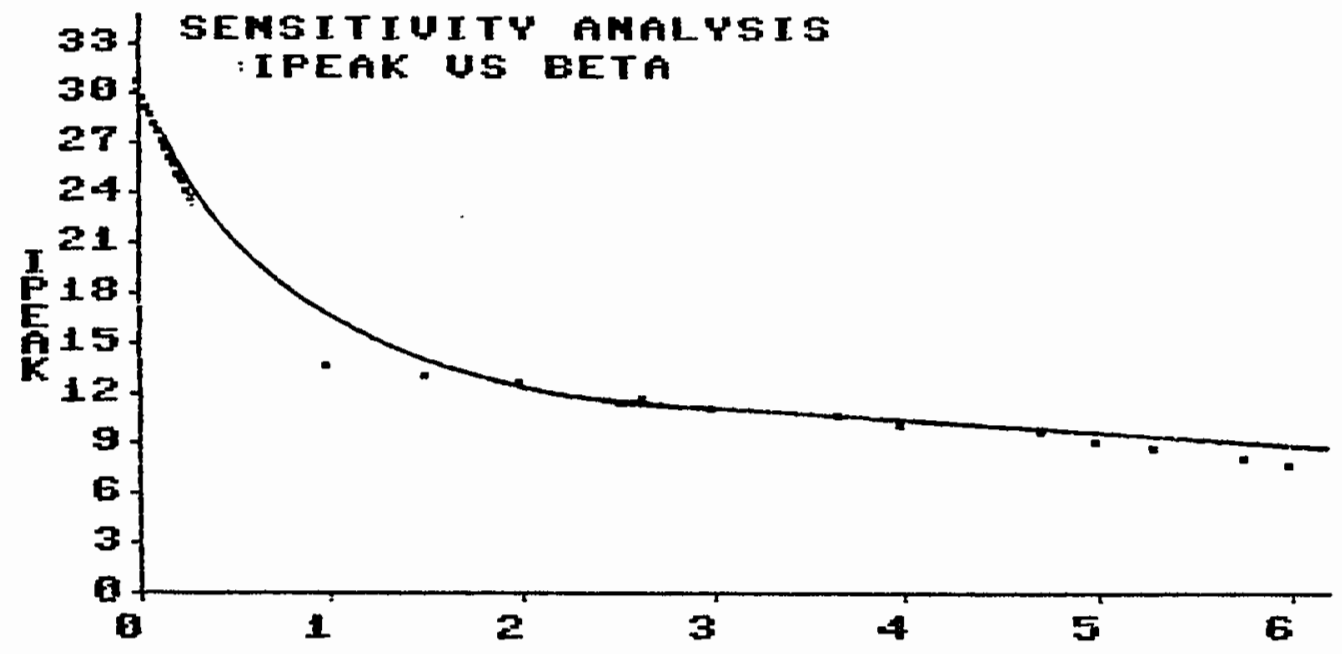

$$
\beta=\left(\ln \left[\max / I_{\min }\right) / a\right.
$$

Figure 19. Peak interigity vo $\beta$. 


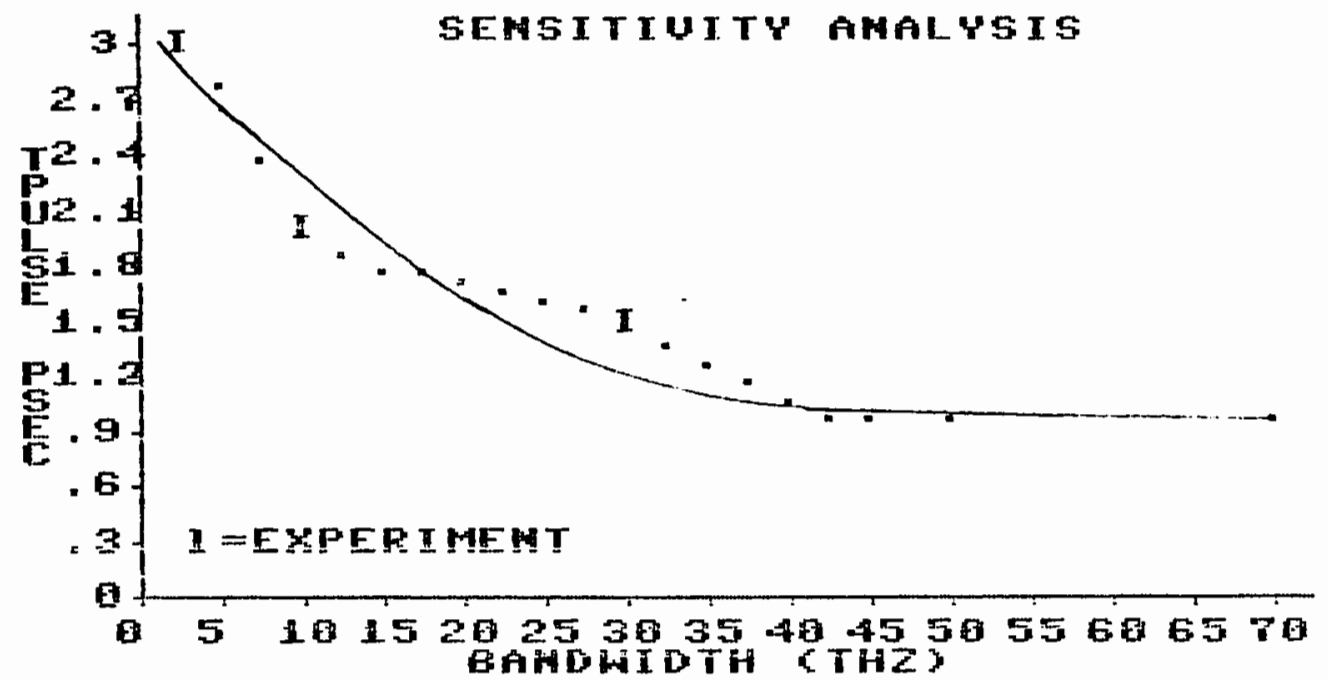

Figure 20. Pulgewidth vs bandwidth. 


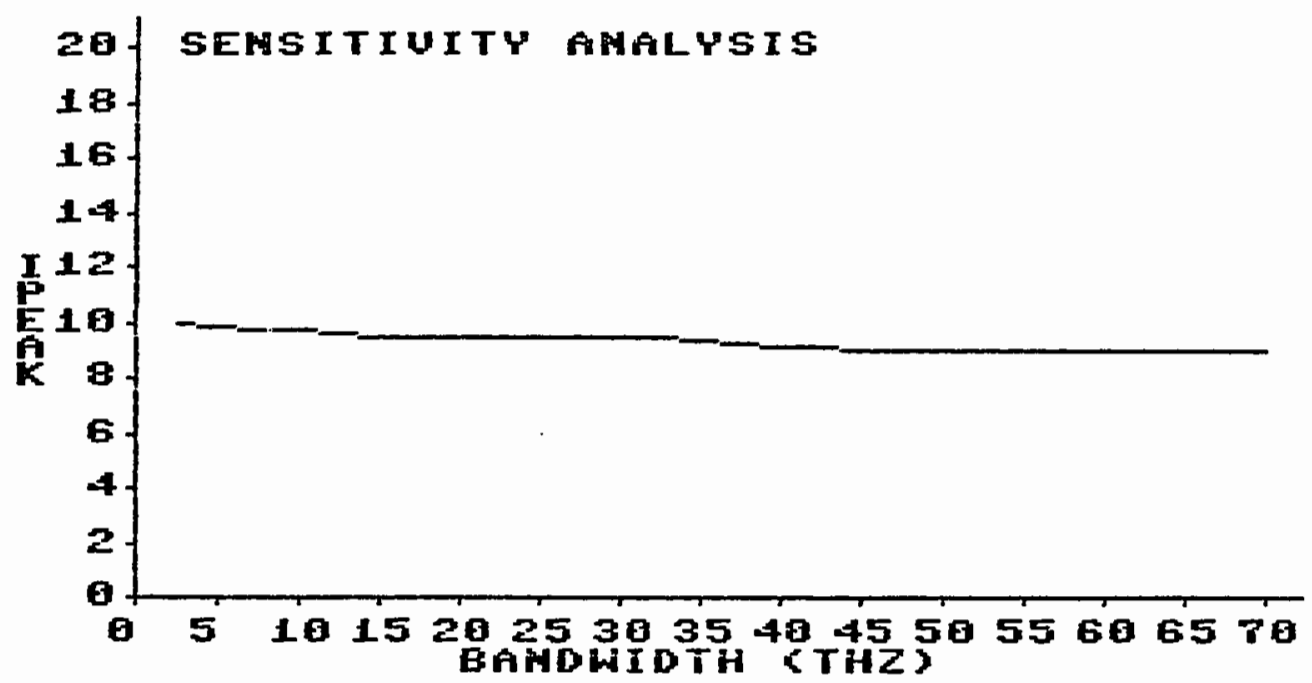

Figure 21. Peak intengity vs bandwidth. 


\section{REFERENCES CITED}

[1] 2.A. Yasa and 0. Teschke, Optics Communication, vol. 15, p. $169,1975$.

[2] A. Scavennec, Optics Communication, vol.17, p. 14, 1976 .

[8] Z.A. Yasa, Applied Physics B, vol. 30, p. 135, 1983.

[4] G.H.C. New and J.M. Cathersl1, Dptics Communication, vol. 50, p. $111,1984$.

[5] W. Heudorfer and G. Marowgky, Applied Physics, vol. 17, p. 181,1978 .

[6] P.G. Kryukov and V. S. Letokhov, Soviet Physics Usp., vol. 12, P. 641, 1970.

[7] D. Faubert and S.L. Chin, Canadian Journal of Physics, vol. 57, p. 1359, 1979.

[8] D.M. KIm, J. Kuhl, R. Lambrich, and D. von der Linde, Optics Communication, vol. 27, p. $123,1978$.

[9] A.E. Siegman and D.J. Kuizenga, Opto-Electron1cs, vol. 6, P. $43,1974$.

[10] C.P. Ausshnitt and R.K. Jain, Applied Physics Letters, vol. 32, p. $727,1978$.

[11] C.P. Ausshnitt, R.K. Ja1n, and J.P. Heritage, IEEE Journal of Quantum Electronics, vol.15, p. $912,1979$.

[12] H.A. Haus, IEEE Journal of Quantum Electronics, val. 11 , P. 736,1975 .

[13] H.A. Haus, IEEE Journal of Quantum Electronics, vol. 11 , P. 323, 1975.

[14] H. A. Haus, Journal of Applied Physics, vol, 46, p. $3049,1975$.

[15] J. Herrmann and U. Motgchmann, Optics Communication, vol. 40, P. 379, 1982 . 
[16] J. Herrmann and U. Motschmann, Applied Physics B, vol. 27 , p. 27,1982 .

[17] L.M. Dayis, J.D. Harvey, and J.M. Peart, Optics Communication, vol. 50, p. $49,1984$.

[18] D. von der Linde, D. Wiechert, and J. Kluge, et al, Proceedings of the Third International Symposium on Ultrafast Processes in Spectrogcopy, Minsk, 1983.

[19] J.M. Catheral, G.H.C. New, and P.M. Radmore, Qptics Letters, vol. 7 , p. 319, 1982 .

[20] L.W. Csaperson, Journal of Applied Physicg, vol. 54, p. $2198,1983$.

[21] V.A. Nekhaenko, S.M. Pershin, and A.A. Podshivalov, Soviet Journal of Quantum Electronicg, vol. 18, no. 3 , p. 299, 1988.

[22] H. Haken, Laser Theory, Springer Verlag, 1989.

[23] W.E. Lamb, Jr., Physical Review A, vol. 134, p. 1429, 1964.

[24] A. Yariv, Quantum Electronics, John wiley \& Sons, 1967.

[25] L.W. Casperson and A. Yariv, IEEE Journal of Quantum Electronics, vol. 8, no. 2, 1972 .

[26] F.P. Schafer, Dye Lasers, Topics in Applied Physics, vol. 1, Springer Verlag, 1973.

[27] G. Holtom and O. Teschke, IEEE Journal of Quantum Electronlcs, vol. 10, no. 8, 1974.

[28] D.R. Preuss and J.L. Gole, Applied Optics, vol. 19, no. $5,1980$.

[29] Spectra-Physics Model 409 Scanning Autocorrelator Manual.

[30] A.J. De Mar1a, et al, IEEE Journal of Quantum Electronlcs, vol. 57, no. 1, p. $2,1989$.

[31] Spectra-Physics Model 375B Dye Laser Manual.

[32] D.C. O'Shea, W.R. Callen and W.T. Rhodes, Introduction to Lasers and Their Applicstions, Addison-Wesley, 1976. 


\section{APPENDIX A}

MECHANISM OF FORMATION OF A PICOSECOND PULSE [21]

To study pulge-ghape evolution in the dye lager with length detuning, we assume that a steady-state regime has been obtained: A narrow pulge is travelling within the dye lager's optical cavity. The ogcillation conditiong require that the dye pulse crosges the amplifier medium when the gain induced by the pump pulse is larger than the intracavity losses. Figure 22 gives a schematic of the variation of the gain due to the passing of the pump and dye pulses through the dye amplifier medium. The horizontal axis is in arbitrary unitg of time at the dye medium. At $t=0$, the pump pulse arrives at the amplifier medium, pumping the dye molecules to the excited state. The gain bullds up following the integral of the pump pulse intensity, until the dye pulse travelling within the cavity reaches the dye amplifier. At this time, a loss of population inversion due to the stimulated emission caused by the dye pulse is induced in the medium. This loss of population shows as a sharp dip in the gain curve at exactly the same time that the dye pulse strikes the amplifier. While the gain drops below the level of the Intracavity losses, the long duration of the pump pulse allows the gain to bulld up again after the dye pulse has passed. 
Figure 22 showg the calculated evolution of the generation of ultrashort pulges. In Figure 22a, the firat argon pump amplifies the dye and generates long pulses here the gain is greater than the losses. In Figure $22 \mathrm{~b}$, the dye amplification lowers gain and narrows the pulse. Figure $22 c$ the amplification saturates andthe pulsewidth gets narrower and Figure $22 d$ is the steady state where the increase in the gain due to pumping is compensated by its reduction due to amplification of the dye pulse. 

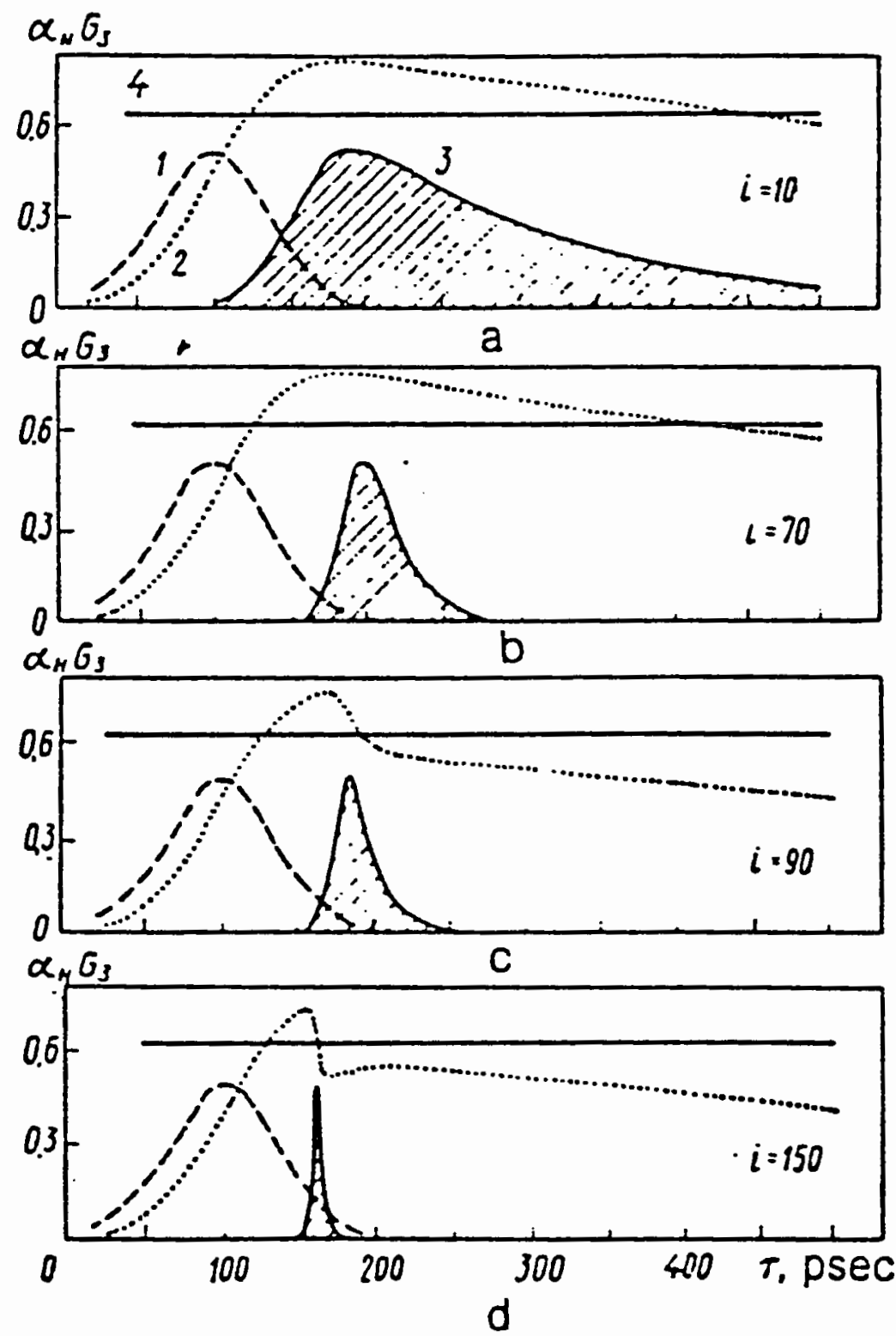

Figure 22. Short pulse formation [21]. 


\title{
APPENDIX B \\ MEASURING PICOSECOND PULSES
}

THE AUTÓCÖFRELATIÓN TECHNIQUE

FOR MEASURING ULTRA-SHORT PULSES [29]

\begin{abstract}
The most common method used for measuring plco-second pulsewidhs is the auto-correlation technique. This technique tranglates differenceg in optical path length into time, uaing the fact that the speed of light is constant. An incoming pulge train is split into two beams of equal intensity. An adjusted optical delay 19 applied to one of the beams and the two beams are then recomilned within a nonlinear cryatal for second harmonic efneration which is proportional to the degree of pulge overlap within the crystal. The intensity of ultraviolet generation as a function of delay between the recombining pulses produces a correlation function related to pulse width. The Spectra-Physics Model 409 Scaning Autocorrelator operates In a background-free conflguration in which the two beams are diaplaced from a common optical axis and recombined in a nencollinear fashion ge in fleure ge. In thie confleuration, the backeround is ellminated becauee ultraviolet 1s generated only at. the point where the two beame intereect with correct phase
\end{abstract}


matching conditions.

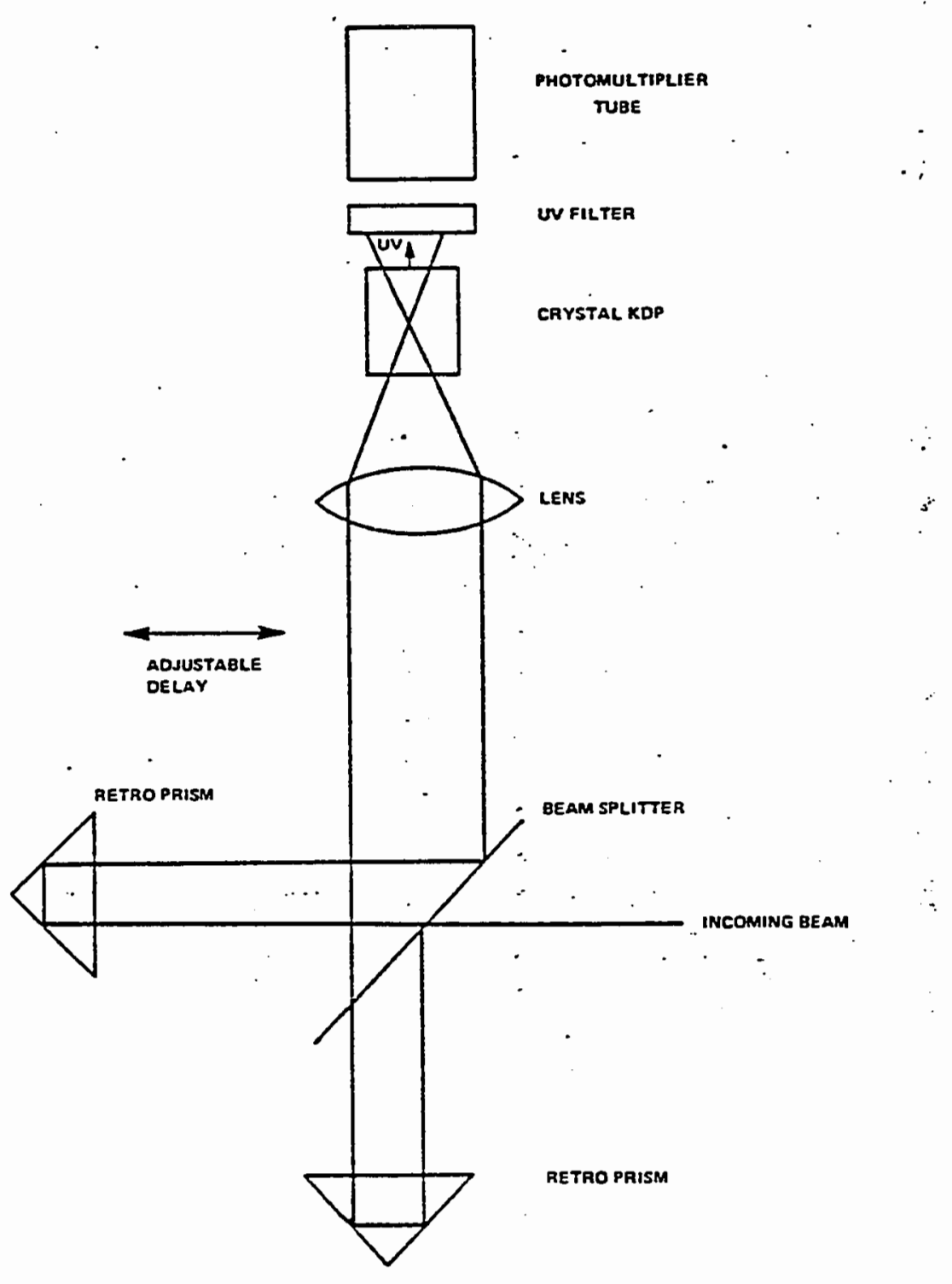

Figure 23. Schematic of Spectra-Physics model 409 autocorrelator [29]. 